\title{
CFD Evaluation of In Situ Probe Techniques for Catalytic Honeycomb Monoliths
}

\author{
Matthias Hettel $^{1}$ - Claudia Antinori ${ }^{2}$ O Olaf Deutschmann ${ }^{1,2}$
}

Received: 16 October 2015 /Revised: 18 May 2016/Accepted: 20 May 2016 /Published online: 4 August 2016

(C) Springer International Publishing Switzerland 2016

\begin{abstract}
Collecting spatially resolved gas phase concentration profiles in catalytic monoliths by applying suction probe techniques has become an important tool for understanding the reaction sequence and for optimizing the design of structured catalysts. The impact of the capillary on the data is investigated by means of computational fluid dynamics (CFD) simulations using the exemplary cases of catalytic partial oxidation of methane over rhodium and oxidation of carbon monoxide over palladium. The influence of a suction probe inside a rectangular channel of a monolith on flow field and concentration profiles are discussed. Four different configurations were investigated: probe in center or corner of the channel with insertion from upstream or downstream in each case. If the capillary is located in the corner of the channel, the influence on the data is negligible. For a position in the center, a noticeable impact was observed. A simple analytical model can predict the reduction of the mass flux due to the insertion of the probe. The worst case exhibits a reduction $>50 \%$. The measuring error is dependent on the direction of insertion of the capillary into the channel (upstream or downstream). Additionally, axial diffusion plays a significant role. For any interpretation of the data, the influence of the probe on the measured data has to be considered. The quantification of the error requires threedimensional CFD simulations.
\end{abstract}

Olaf Deutschmann

deutschmann@kit.edu

1 Institute for Chemical Technology and Polymer Chemistry, Karlsruhe Institute of Technology (KIT), Kaiserstr. 12, 76128 Karlsruhe, Germany

2 Institute of Catalysis Research and Technology, Karlsruhe Institute of Technology (KIT), Kaiserstr. 12, 76128 Karlsruhe, Germany
Keywords CFD - Suction probe technique - Spatial profile sampling · In-situ measurement · Catalytic monolith

\section{Introduction}

The in-depth understanding of processes for optimization of operating conditions and catalyst design is challenging. In many cases, the interaction of chemical reaction and mass and heat transfer leads to a complex system, which needs to be understood to make optimization possible. To gain a deeper insight, in situ sampling techniques for measuring spatially resolved concentration and temperature profiles become more and more popular. There is a wide variety of techniques, invasive and non-invasive [1,2]. For structured catalyst, e.g., honeycomb monoliths and foams, often capillary-based techniques are chosen. The measuring devices for analysis of the gas samples differ as does the diameter used. In these invasive techniques, the detected profiles are influenced by the presence of the capillary. The techniques are applied to different research areas, such as exhaust gas after treatment [3-6] and catalytic partial oxidation of hydrocarbon fuels [7-9]. The operating conditions at which the sampling techniques are applied differ in operating conditions, e.g., temperature (473-573 K for $\mathrm{NO}_{\mathrm{x}}$ removal [10]; $>1000 \mathrm{~K}$ for catalytic partial oxidation (CPOX) [7]) and gas composition. Thus, the influence of the capillary on the detected spatially resolved data could differ.

In the present work, the impact of the probe of an in situ measuring equipment on the measured data is investigated by means of computational fluid dynamics (CFD) simulations. The aim of the work is to understand the influence of the probe on the spatial profiles of the species concentrations, which is important for the interpretation of the data and the design of the experimental setup. 
In our recent CFD study of the CPOX of methane over Rh, coated honeycombs already revealed the potential offset of the axial concentration profiles measured, which is dependent on the radial and axial position of the probe in the monolith channel [11]. With respect to the radial position, the influence is the largest for positioning the probe in the center and the smallest for the corner position. For both configurations, the influence of the probe obviously increases, the deeper the probe is inserted into the channel. In general, the presence of the probe leads to an increasing residence time of the reactants inside the channel. The impact on the data can be corrected after the measurement, if the temperature gradient along the channel is small and the probe is located in the channel center [11].

Many fundamental questions concerning the influence of the probe are unanswered. In our experimental studies $[5,9,11,12]$, the probe is launched into the monolith from the front side. This configuration was chosen to avoid the hot temperature region downstream of the catalyst (for CPOX approx. $950 \mathrm{~K}$ ) as reactions inside the probe might occur. Other groups (e.g., [4, 13]) prefer to suck from the back side of the monolith.

In this work, the influence of direction of insertion of the capillary is discussed. A system without chemical reaction and two with, namely, the catalytic partial oxidation of methane (CPOX) over rhodium and the oxidation of carbon monoxide (CO-OX) over palladium, are considered. For each case, the results of the CFD simulations are compared to experimental data. The focus of this work is the evaluation of the influence of the probe on the measured data. The validation of the reaction mechanisms used is beyond the scope of this work.

\section{Experimental Setup}

A sketch of the reactor setup and of the sampling technique used for measuring the concentration and temperature profiles inside a monolith is given in Fig. 1 for a

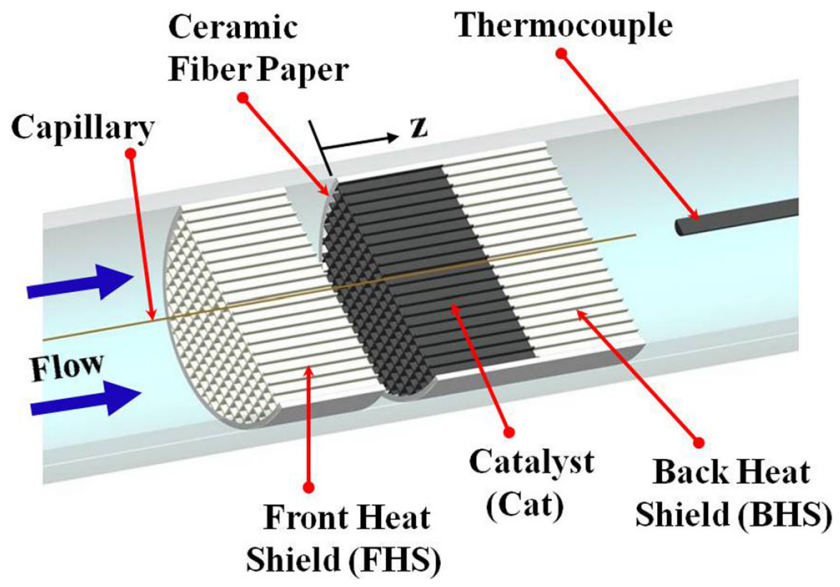

Fig. 1 Sketch of the experimental system [9] capillary inserted from the upstream (front) side. A coated monolithic honeycomb catalyst $(600 \mathrm{cpsi}$, length $=10 \mathrm{~mm}$, diameter $=19 \mathrm{~mm}$ ) is placed inside a quartz glass tube with uncoated monoliths (600 cpsi) at both sides. These act as heat shields to diminish the heat loss due to radiation and serve additionally as fixations for the suction probe and the thermocouple. A gap of $5 \mathrm{~mm}$ was kept between the front heat shield (FHS) and catalyst to avoid flow disturbance at the catalyst entrance. The back heat shield (BHS) is positioned directly after the catalyst. The reactor is placed inside a furnace to ignite the reaction and to control the temperature.

The CPOX of methane was studied over a rhodium/ alumina monolith (Delphi Catalyst Inc., catalyst loading $4.23 \mathrm{mg} / \mathrm{cm}^{3}$, washcoat $\gamma-\mathrm{Al}_{2} \mathrm{O}_{3}$, thickness $100 \mu \mathrm{m}$ ) at a molar $\mathrm{C} / \mathrm{O}$ ratio of unity in a dilution of $80 \mathrm{vol} \%$ nitrogen. The $\mathrm{C} / \mathrm{O}$ ratio is defined as the ratio of the molar amount of carbon atoms in methane and the molar amount of oxygen atoms in molecular oxygen entering the reactor. The mixture was preheated to $363 \mathrm{~K}$. The oven temperature was fixed at $523 \mathrm{~K}$.

For the investigation of the $\mathrm{CO}-\mathrm{OX}$, a palladium/aluminacoated monolith (Umicore AG \& Co. KG, catalyst loading $1.06 \mathrm{mg} / \mathrm{cm}^{3}$, washcoat $\gamma-\mathrm{Al}_{2} \mathrm{O}_{3}$, thickness $40 \mu \mathrm{m}$ ) was used. A mixture of 1087 ppm $\mathrm{CO}$ and $4.9 \% \mathrm{O}_{2}$ in nitrogen was provided and preheated to $363 \mathrm{~K}$. The oven temperature was fixed at $463 \mathrm{~K}$.

The volumetric fluxes used in the experiments can be found in Table 1. Concentration profiles were detected by continuously sucking a small amount of gas $(2 \mathrm{ml} /$ min) into the capillary having an outer diameter of $170 \mu \mathrm{m}$. Element balances (C, H, O) typically closed within $\pm 5-10 \%$. The capillary was moved along the channel by a motorized linear stage. The axial profile of the gas phase temperature was determined by a thermocouple and the wall temperature by an optical fiber connected to an infrared narrow band pyrometer. The measurements of the concentration and the temperature

Table 1 Boundary conditions

\begin{tabular}{llllll}
\hline & & $\mathrm{a}$ & $\mathrm{b}$ & $\mathrm{c}$ & $\mathrm{d}$ \\
& & $\begin{array}{l}\text { No } \\
\text { reaction }\end{array}$ & $\begin{array}{l}\text { Reaction } \\
\text { CPOX, } \\
\mathrm{T}_{\text {wall }}=\text { const. }\end{array}$ & $\begin{array}{l}\text { Reaction } \\
\text { CPOX, } \\
\mathrm{T}_{\text {wall }}=\mathrm{f}(\mathrm{z})\end{array}$ & $\begin{array}{l}\text { Reaction } \\
\text { CO-OX } \\
\mathrm{T}_{\text {wall }}=\text { const. }\end{array}$ \\
\hline & & & 0.218 & 0.218 & 0.277 \\
$\dot{V}_{\text {inlet }}$ & {$\left[\mathrm{m}^{3}{ }_{\mathrm{N}} / \mathrm{h}\right]$} & 1.020 & 0.218 & \\
$u_{\text {inlet }}$ & {$[\mathrm{m} / \mathrm{s}]$} & 1.000 & 0.680 & 0.680 & 0.460 \\
$T_{\text {inlet }}$ & {$[\mathrm{K}]$} & 300 & 873 & 873 & 463 \\
$T_{\text {wall }}$ & {$[\mathrm{K}]$} & 300 & 973 & Experiment & 463 \\
$\mathrm{X}_{\mathrm{CH} 4}$ & {$[-]$} & 0.133 & 0.133 & 0.133 & 0.000 \\
$\mathrm{X}_{\mathrm{CO}}$ & {$[-]$} & 0.000 & 0.000 & 0.000 & 0.0011 \\
$\mathrm{X}_{\mathrm{O} 2}$ & {$[-]$} & 0.067 & 0.067 & 0.067 & 0.0489 \\
$\mathrm{X}_{\mathrm{N} 2}$ & {$[-]$} & 0.800 & 0.800 & 0.800 & 0.9500 \\
\hline
\end{tabular}


profiles were conducted one by one. A more detailed explanation of the experimental procedures can be found in $[9,11,12]$.

\section{Modeling Approach}

\subsection{Computational Tool}

The CFD code FLUENT [14] was applied for the numerical simulation of the three-dimensional flow field. The secondorder upwind scheme was applied for the discretization of the convective terms. The determination of the species properties was based on the kinetic theory.

\subsection{Reaction Kinetics}

The mean field approximation was applied for modeling the surface chemistry $[15,16]$. The total molar production rate $s_{\mathrm{i}}$ of a gaseous species, an adsorbed surface species, or a bulk species due to surface reactions is calculated in analogy to gas phase reactions as a product of rate coefficients and concentrations determined by

$\dot{s}_{\mathrm{i}}=\sum_{\mathrm{k}=1}^{K_{s}} v_{\mathrm{ik}} k_{\mathrm{fk}} \prod_{\mathrm{i}=1}^{N_{\mathrm{g}}+N_{\mathrm{s}}+N_{\mathrm{b}}} c_{\mathrm{i}}^{v_{\mathrm{ik}}^{\prime}} \quad\left(i=1, \ldots, N_{\mathrm{g}}+N_{\mathrm{s}}+N_{\mathrm{b}}\right)$.

The mechanism consists of $K_{s}$ surface reactions (including adsorption and desorption) among $N_{\mathrm{g}}$ gas phase, $N_{\mathrm{s}}$ surface, and $N_{\mathrm{b}}$ bulk species. $\nu_{\mathrm{ik}}$ is the stoichiometric coefficient, and $c_{\mathrm{i}}$ is the species concentration for $N_{\mathrm{s}}$ adsorbed species and for the $N_{\mathrm{g}}$ and $N_{b}$ gaseous and bulk species, respectively. The rate coefficients $k_{\mathrm{fk}}$ can be described by an Arrhenius model.

The concentration of the absorbed species can be expressed in terms of a surface coverage $\Theta_{i}$ according to the relation

$\Theta_{\mathrm{i}}=\frac{c_{\mathrm{i}} \cdot \sigma_{\mathrm{i}}}{\Gamma}$

where the concentration $c_{\mathrm{i}}$ is given in moles per square meter for the adsorbed species and moles per cubic meter for gaseous and bulk species. $\sigma_{\mathrm{i}}$ is the number of surface sites that are occupied by species i, and $\Gamma$ is the surface site density, i.e., the number of adsorption sites per catalytic surface area. Thus, locally resolved reaction rates depend on the local gas phase concentrations, surface coverage, and temperature.

The flux of species $j_{\mathrm{i}, \mathrm{s}}\left(\right.$ in $\mathrm{mol} / \mathrm{m}^{2}$ ) on the outer surface of the washcoat is given by

$j_{\mathrm{i}, \mathrm{s}}=\dot{s}_{\mathrm{i}} \cdot M_{\mathrm{i}} \cdot F_{\text {cat } / \mathrm{geo}} \cdot \eta_{\mathrm{i}}$

where $s_{\mathrm{i}}$ is the surface reaction rate of species i, $M_{\mathrm{i}}$ is the molecular weight of species $\mathrm{i}$, and $F_{\text {cat/geo }}$ is the ratio of catalytically active surface area to geometric surface area. The detailed consideration of diffusion effects inside the washcoat is complicated (e.g., [17]) and beyond the scope of this work. Therefore, internal mass transfer limitations are taking into account by means of an effectiveness factor $\eta_{\mathrm{i}}$. The effectiveness factor is the ratio of the observed reaction rate to that which would occur in case of no internal diffusion limitations. For both reaction mechanisms used, we performed additional 2D calculations with and without washcoat models to estimate $\eta_{\mathrm{i}}$. For this, we applied the DETCHEM ${ }^{\mathrm{TM}}$ tool CHANNEL, where a spherical channel is discretized in two dimensions (axial and radial direction). The product $F_{\text {cat/geo }} \cdot \eta_{\mathrm{i}}$ is used in the CFD code to simultaneously account for active catalytic surface area and internal diffusion limitation in the washcoat.

\subsubsection{Catalytic Partial Oxidation of Methane on Rhodium}

A multi-step surface reaction mechanism was used, which was developed for the partial oxidation and autothermal reforming of methane over rhodium [18]. The mechanism includes 7 gas phase species, 23 surface species, and 38 reactions. For the rhodium-coated honeycomb catalyst, a value of $F_{\text {catgeo }}=185$ was calculated based on the measured dispersion. The beforementioned $2 \mathrm{D}$ calculations led to a value of $\eta_{\mathrm{i}}=0.027$, which was kept constant in the CFD simulations.

It was already shown in literature that reactions in the gas phase can be neglected for the given operating conditions, which was also confirmed experimentally and computationally by our studies [19-22] (not further discussed herein).

\subsubsection{Oxidation of Carbon Monoxide on Palladium}

A multi-step surface reaction mechanism was used, which includes 3 gas phase species, 5 surface species, and 10 reactions [23]. For the palladium catalyst, the measured dispersion resulted in $F_{\text {cat/geo }}=33$. With the preceding $2 \mathrm{D}$ calculations, a value of $\eta_{\mathrm{i}}=0.318$ was deduced. Reactions in the gas phase can surely be neglected at the low temperature of $463 \mathrm{~K}$.

\subsection{Computational Domains and Grids}

The computations were performed for a monolithic honeycomb with $600 \mathrm{cpsi}$. The two calculation domains each include nine partially sliced channels (one of them with probe) of the coated monolith and a flow region with the length of $10 \mathrm{~mm}$ upstream and downstream (Fig. 2). The FHS and BHS were not taken into account. The solid material of the catalyst was not included in the calculation domains. For simplicity, we neglected the rounding of the channel edges due to the washcoat. The free height of a single quadratic channel is $H=795 \mu \mathrm{m}$. Diffusion through the channel walls was neglected. 
The probe points into the $z$-direction and is perpendicular to the $x y$-plane. The outer diameter of the probe is $D_{\text {probe,o }}=170 \mu \mathrm{m}$ and the inner diameter is $D_{\text {probe,I }}=100 \mu \mathrm{m}$. We compared four different basic configurations for the positioning of the probe.

The sketches center-upstream and center-downstream in Fig. 2 show the calculation domains of the configurations where the probe is placed in the center of the channel and enters the channel from upstream and from downstream, respectively. The pictures corner-upstream and cornerdownstream show the calculation domains of the setups in which the probe is placed in the corner of the channel and enters the channel from upstream and from downstream, respectively. In all four sketches, the probe tip is at $z_{\text {probe }}=5 \mathrm{~mm}$.

In each picture, the inflow is on the left side (red) and the outflow is on the right side (blue). Part of the fluid is sucked in at the tip of the probe, which is colored dark blue. Symmetry conditions were applied at all surfaces of the computational domains normal to the $x$ - and $y$-directions (yellow). Thus, the calculation domain including the probe repeats virtually into the $x$ - and $y$-directions. Figures 3 and 4 show a cut through the $x y$-plane of the two configurations (probe in the center or in the corner of a channel), which depict this behavior. The solid walls of the catalyst are colored green, the flow region is colored red, and the capillary is colored blue.

Only a quarter of the probe is inside the calculation domain for the configurations in which the probe is in the center of a channel (zoom in Fig. 3). The shape of the probe was slightly varied (zoom in Fig. 4) for the configurations, in which the probe is in the corner of a channel, to allow the generation of a block-structured hexahedral grid [24].

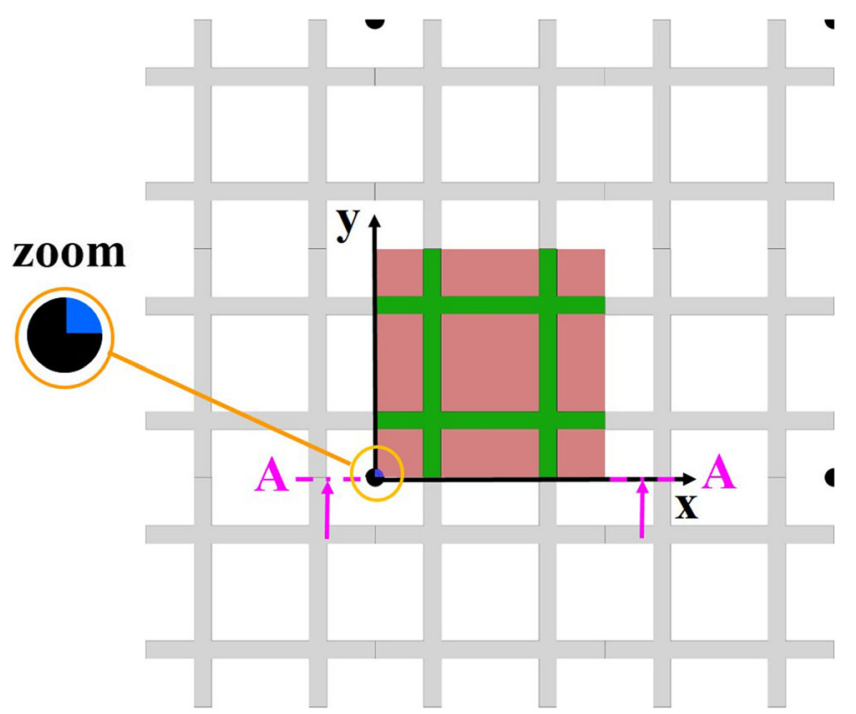

Fig. 3 Probe is in the center of the channel. Cut through the $x y$-plane of the calculation domain. The grey regions of the monolith depict the far field of the calculation domain resulting from the symmetry conditions. The enlarged section shows the shape of the probe, of which the blue portion is inside the calculation domain. (Color figure online)

The conditions in the channel with the probe (subscript probe) and in a reference channel without probe (subscript ref) are compared (Fig. 2). The reference channel is the channel without probe, which is the farthest from the channel with probe and is therefore experiencing the smallest influence of the probe. In general, the difference of the volumetric fluxes in the various empty channels has been shown to be very small [24].

For each of the four configurations, nine axial positions of the probe tip $\left(z_{\text {probe }}=0 / 1.25 / 2.5 / 3.75 / 5 / 6.25 / 7.5 / 8.75 / 10 \mathrm{~mm}\right)$ were simulated (Fig. 5). The position of the probe tip in the $z$ -
Fig. 2 Calculation domains. Center-upstream: probe in the center entering from upstream, center-downstream: probe in the center entering from downstream, corner-upstream: probe in the corner entering from upstream, corner-downstream: probe in the corner entering from downstream

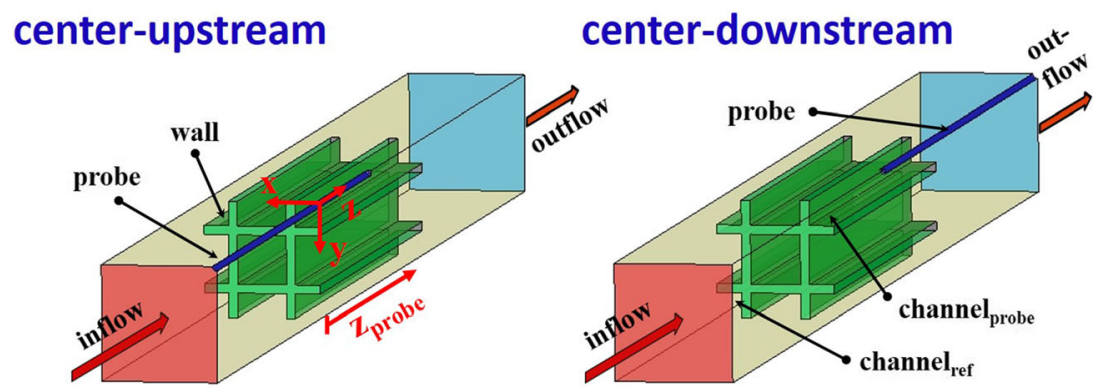

corner-upstream
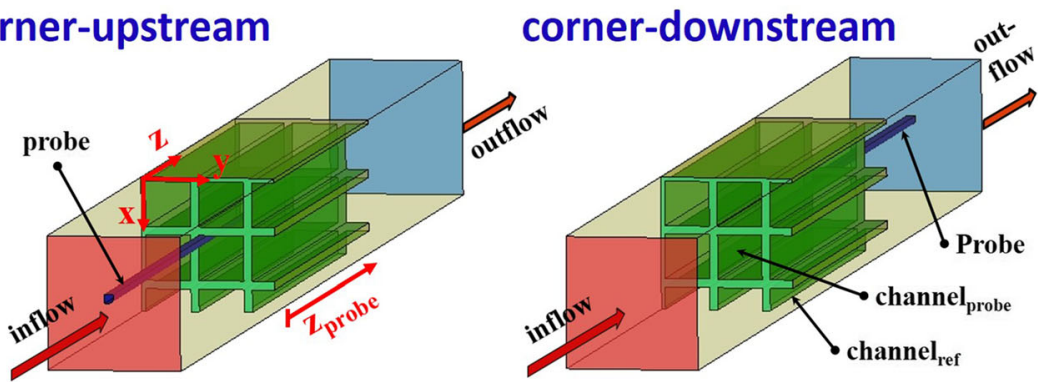


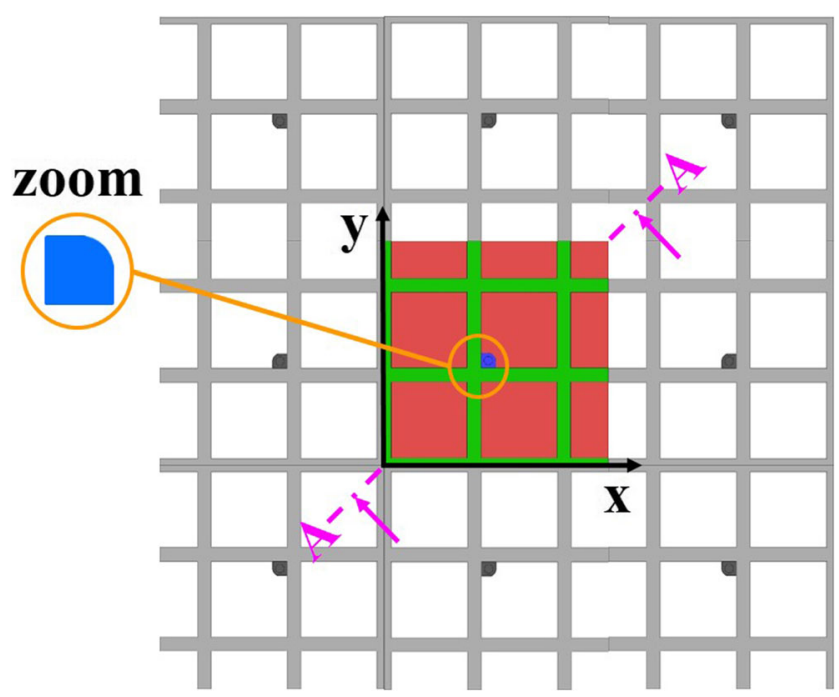

Fig. 4 Probe is in the corner of the channel. Cut through the $x y$-plane of the calculation domain. The grey regions of the monolith depict the far field of the calculation domain resulting from the symmetry conditions. The enlarged section shows the shape of the probe. (Color figure online)

direction is always measured from the upstream surface of the monolith downstream into the positive $z$-direction. For each position of the probe tip in axial and radial direction, a blockstructured grid with a size of approx. 2,000,000 prism cells was generated. The typical discretization of the flow region inside a single channel with a length of $10 \mathrm{~mm}$ was 250 nodes in the $z$-direction and 30 nodes into the directions $x$ and $y$.

\subsection{Inlet and Boundary Conditions}

The inlet and boundary conditions used for the CFD calculations are listed in Table 1.

The velocity $u_{\text {inlet }}$ of the gas mixture at the inlet of the computational domain calculates from the volumetric flux $\dot{V}_{\text {inlet }}$ given in the experiments, the measured temperature $T_{\text {inlet }}$ of the mixture, and the cross-sectional area of the reactor tube $($ diameter $=19 \mathrm{~mm})$.

For the calculation of the flow without chemical conversion, all temperatures were set to $300 \mathrm{~K}$ (boundary case "a"). Two different conditions for $T_{\text {wall }}$ have been used for the CPOX. In case "b," an estimated wall temperature of $973 \mathrm{~K}$ was used. In case "c," the experimentally determined wall temperature profile

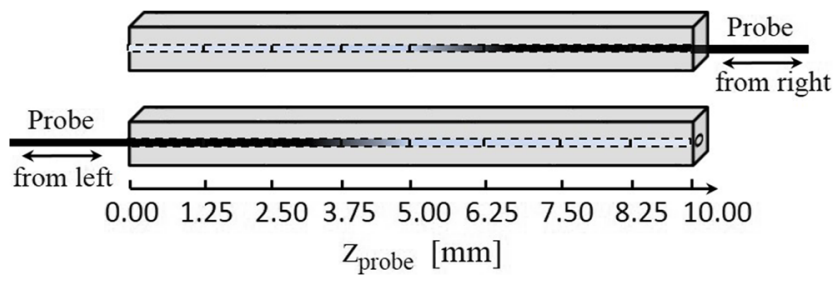

Fig. 5 Axial positions of the probe tip, $z_{\text {probe }}$, examined in the calculations was used. In both cases, we applied the temperature, which was measured directly downstream of the front heat shield, as the inlet temperature of the mixture, $T_{\text {inlet }}=873 \mathrm{~K}$. The mole fractions $X$ of the gas phase species were chosen according to the experimental operating conditions (Sect. 2).

For the CO oxidation (case "d"), both the temperature at the inlet, $T_{\text {inlet }}$, and the temperature, $T_{\text {wall }}$, at the surfaces of the solid material were set to $463 \mathrm{~K}$ (oven temperature). The gas composition was chosen based on the experimental operating conditions (Sect. 2).

The pressure at the outflow boundary of the calculation domains, $p_{\text {outlet }}$, was set to be 1.0 bar. The Reynolds number of the flow in the channels was always lower than 80 , and thus, the flow was laminar.

\section{Analytical Model}

A simple analytical model is presented to estimate the reduction of volumetric flux in case of a probe in the center of the channel at isothermal conditions (Fig. 6). We start from the formula for the pressure loss of a fully developed laminar flow in a circular channel [25]:

$$
\Delta p=p_{\text {outlet }} p_{\text {inlet }}=\frac{\rho}{2} \cdot u^{2} \cdot \frac{L}{D} \cdot \lambda_{\text {laminar }} \quad \text { with } \quad \lambda_{\text {laminar }}=\frac{\mathrm{C}}{R e} .
$$

Here, $\rho$ is the density, $u$ the radially averaged axial velocity, $D$ the channel diameter, and $L$ the length of the channel. The loss coefficient $\lambda_{\text {laminar }}$ includes the Reynolds number $R e=u \cdot D / v$, where $v$ is the kinematic viscosity. For non-circular channels, the hydraulic diameter $D_{\text {hyd }}$ is used instead of $D$ [25]:

$D_{\text {hyd }}=\frac{4 \cdot A}{U}$

where $A$ is the cross-sectional area of a tube of arbitrary shape and $U$ is the length of the interface between the solid wall and the fluid in this area (wetted perimeter). This approach makes it possible to apply Eq. (4) for non-spherical tubes. The constant $C$ depends on the shape of the tube. The application of Eq. (5) yields the following hydraulic diameters for a channel with and without probe, respectively:

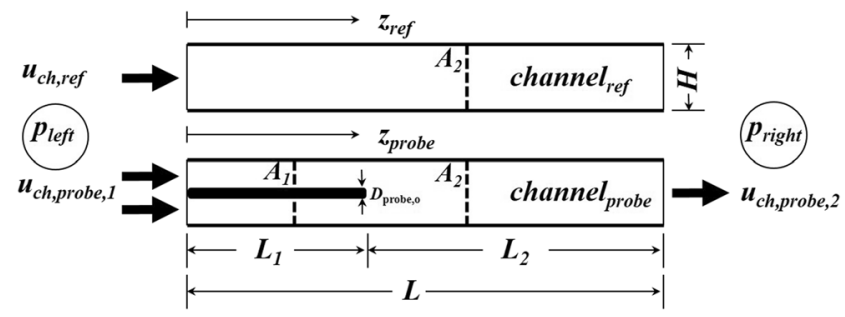

Fig. 6 Geometry and symbols used for the analytical model 
$D_{\text {hyd,ch,ref }}=\frac{4 \cdot H^{2}}{4 \cdot H}=H$

$D_{\text {hyd,ch,probe }}=\frac{4 \cdot\left(H^{2}-\pi / 4 \cdot D_{\text {probe }, \mathrm{o}}^{2}\right)}{\left(4 \cdot H+\pi \cdot D_{\text {probe }, \mathrm{o}}\right)}$

The height of the quadratic channel is $H=795 \mu \mathrm{m}$, and the outer diameter of the probe is $D_{\text {probe, }}=170 \mu \mathrm{m}$. The indices ch,ref and ch,probe represent the values in a reference channel (without probe) and in the channel with probe, respectively.

We start from the assumption that the flow upstream and downstream of the monolith is laminar and incompressible. Additionally, the velocity into directions perpendicular to the $z$-axis upstream and downstream of the monolith is assumed to be small against the axial velocity. Based on these assumptions, the flow can be described by the boundary equations and no radial gradient of the pressure can be found upstream and downstream of the monolith. Therefore, the pressure loss is the same in all channels.

The pressure loss in the channel with probe is the sum of two parts. The first channel section with the length $L_{1}$ (probe tip at $L_{1}=z_{\text {probe }}$ ) leads to the loss $\Delta p_{\text {ch,probe, } 1 \text {. The second }}$ section with the length $L_{2}=\left(L-L_{1}\right)$ leads to the loss $\Delta p_{\mathrm{ch}}$, probe,2. Therefore, we can write

$$
\begin{aligned}
\Delta p & =p_{\text {outflow }}-p_{\text {inflow }}=\Delta p_{\text {ch,ref }}=\Delta p_{\text {ch,probe }} \\
& =\Delta p_{\text {ch,probe }, 1}+\Delta p_{\text {ch,probe }, 2}
\end{aligned}
$$

Division by the last term, substitution with Eq. (4), and insertion of the hydraulic diameters from Eqs. (6) and (7) yield

$$
\begin{gathered}
\frac{\Delta p_{\text {ch,ref }}}{\Delta p_{\text {ch,probe }, 1}+\Delta p_{\text {ch,probe }, 2}}=1= \\
\frac{u_{\text {ch,ref }} \cdot C \cdot \frac{L}{D_{\text {hyd,ch,ref }}{ }^{2}}}{u_{\text {ch,probe }, 1} \cdot C_{1} \cdot\left(\frac{L_{1}}{D_{\text {hyd,ch,probe }}{ }^{2}}\right)+u_{\text {ch,probe }, 2} \cdot C_{2} \cdot\left(\frac{L_{2}}{D_{\text {hyd,ch,ref }}{ }^{2}}\right)}
\end{gathered}
$$

The volumetric flux inside the channel with probe has to be the same in the two sections with and without probe (isothermal conditions); hence,

$$
\begin{aligned}
\dot{V} & =\text { constant }=\dot{V}_{\text {ch,probe }, 1}=\dot{V}_{\text {ch,probe }, 2}=A_{1} \cdot u_{\text {ch,probe }, 1} \\
& =A_{2} \cdot u_{\text {ch,probe }, 2}
\end{aligned}
$$

where $A_{1}$ is the cross-sectional area of the part of

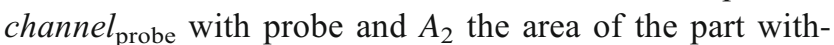
out probe (see Fig. 6). Equation (11) leads to a relation between the two velocities $u_{\text {ch,probe, } 1}$ and $u_{\text {ch,probe, } 2}$ in channel $_{\text {probe: }}$

$$
u_{\text {ch,probe }, 1}=u_{\text {ch,probe }, 2} \cdot \frac{A_{2}}{A_{1}}
$$

By inserting Eq. (11) into Eq. (9) and rearranging, we get a relation for the ratio of the volumetric fluxes in the channels with and without probe, which is the same as the ratio of the velocities $u_{\text {ch,probe, } 2}$ and $u_{\text {ch,probe, } 1}$ :

$$
\begin{aligned}
\frac{\dot{V}_{\text {ch,probe }}}{\dot{V}_{\text {ch, ref }}} & =\frac{u_{\text {ch,probe }, 2}}{u_{\text {ch,ref }}} \\
& =\frac{C \cdot \frac{L}{D_{\text {hyd,ch,ref }}{ }^{2}}}{\left(\frac{A 2}{A 1}\right) \cdot C_{1} \cdot\left(\frac{L_{1}}{D_{\text {hyd,ch,probe }}^{2}}\right)+C_{2} \cdot\left(\frac{L_{2}}{D_{\text {hyd,ch,ref }}^{2}}\right)}
\end{aligned}
$$

As mentioned above, the constant $C$ in the relation $\lambda_{\text {laminar }}=\mathrm{C} / \operatorname{Re}$ depends on the form of the tube. For a square tube (empty channel and empty section of channel with probe), the value is $C=C_{1}=64$. For the channel section with probe (cylinder in square-shaped channel), no formulas for the constant $C$ can be found in literature. Therefore, we assumed here a spherical geometry (cylinder in spherical channel) with diameter $D=H$. The ratio of these measures is $D / D_{\text {probe, o }}=795 \mu \mathrm{m} / 170 \mu \mathrm{m} \approx 5$. From this proportion, we can deduce a value of $C_{1}=\mathrm{f}\left(D / D_{\text {probe,o }}\right)=93$ [26]. A new approach to evaluate exact friction factor correlations for rectangular channels with interior cylindrical bodies can be found in [27].

The residence time of the mixture at position $z_{\text {probe }}$ in channel $_{\text {probe }}$ is larger than the residence time at the same axial position $z_{\text {ref }}$ in channel $_{\text {ref. }}$ The larger the residence time, the further is the reaction progress. We search now for the distinct position $z$ in channel $_{\text {ref }}$, for which the residence time $\tau_{\text {ref }}$ is the same as the residence time $\tau_{\text {probe }}$ at the position $z_{\text {probe }}$ in channel $_{\text {probe }}$. The residence time for each of the two positions can be determined by

$\tau_{\text {ref }}=\tau_{\text {probe }}=\frac{z_{\text {probe }}}{u_{\text {ch,probe }, 2}}=\frac{z}{u_{\text {ch,ref }}}$

From this formula, we get the axial position $z$ in channel $_{\text {ref }}$ in dependency of the position of the probe tip $z_{\text {probe }}$ in channel $_{\text {probe }}$ and the ratio of velocities (Eq. 12):

$z=\frac{z_{\text {probe }}}{\frac{u_{\text {ch,probe }, 2}}{u_{\text {ch,ref }}}}$

If the two positions $z$ and $z_{\text {probe }}$ exhibit the same residence time, it can also be expected that the reaction progress, and beyond that, also the species concentrations are the same. That means that the concentration values which would be measured with the probe at an axial position $z_{\text {probe }}$ would belong to the axial position $z$ in an empty channel [11]. 
The simplifications of the analytical model are as follows: The flow is instantaneously developed in the channel. The parts of the probe outside the channel are not considered. There, a boundary layer develops at the outer surface of the probe.

\section{Results and Discussion}

In Sect. 5.1, the influence of the probe on the flow field is discussed. Additionally, the analytical model is compared to the CFD simulations. In Sect. 5.2, the influence of the probe on the concentration profiles is examined by comparison of calculated and measured data.

\subsection{Influence of Probe on Flow Field}

\subsubsection{Calculation Without Reaction}

All results shown in this section are based on the boundary conditions of case a in Table 1.

Firstly, the flow field of configuration center-upstream will be discussed. Figure 7 shows isoplots of the velocity into the $z$-direction in the $y z$-plane through the center of the channels (cross section A-A given in Fig. 3) for three different positions of the probe tip (from top to bottom: $z_{\text {probe }}=0 / 5 / 10 \mathrm{~mm}$ ). Only one quarter of channel $_{\text {probe }}$ needs to be computed. The white blocks indicate the solid parts of the monolith, and the black block indicates the probe. The flow direction is from left to right. The Reynolds number in the channel is $R e \approx 80$.

As expected, the velocity in the channel with probe is smaller the deeper the probe is launched into the channel.

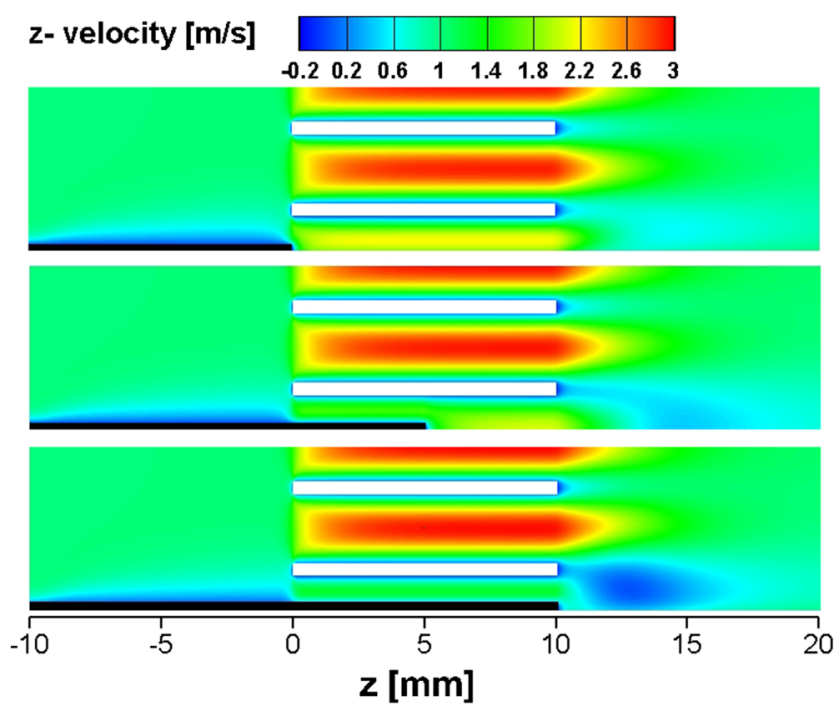

Fig. 7 Configuration center-upstream. Z-velocity in the $y z$-plane (view A-A in Fig. 3) for three positions $z_{\text {probe }}=0 / 5 / 10 \mathrm{~mm}$ of the probe tip (from top to bottom). The scale of the plots is compressed in the $z$-direction by a factor 3
An interesting fact is that the probe influences a region downstream of channel $_{\text {probe. }}$ As the volumetric flux in channel $_{\text {probe }}$ is smaller than in the other channels, a region with lower velocity can be identified behind channel $l_{\text {probe }}$. This wake region is more pronounced for probes farther inserted into the channel.

In Fig. 8, the velocity into the $z$-direction is shown for the configuration center-downstream for three different positions of the probe tip (from top to bottom: $z_{\text {probe }}=10 / 5 / 0 \mathrm{~mm}$ ). For the same depth of immersion of the probe, the results are very similar to the configuration center-upstream. For example, the flow field of configuration center-upstream with $z_{\text {probe }}=10 \mathrm{~mm}$ is qualitatively similar to the flow field of configuration center-downstream with $z_{\text {probe }}=0 \mathrm{~mm}$ (depth of immersion in both cases $10 \mathrm{~mm}$ ). Again, a region with lower velocity can be identified downstream of channel $_{\text {probe }}$.

The ratio of the volumetric fluxes in a channel with probe and a channel without probe $\dot{V}_{\text {channel,probe }} / \dot{V}_{\text {channel,ref }}$ is shown in Fig. 9 for the configurations center-upstream and centerdownstream. The deeper the probe is inserted into the channel, the smaller is the volumetric flux in channel $_{\text {probe }}$. For centerupstream with $z_{\text {probe }}=0 \mathrm{~mm}$, the volumetric flux in channel $_{\text {probe }}$ reduces to approx. $87 \%$ compared with the flux in channel $_{\text {ref. }}$. This reduction is an effect of the boundary layer formed at the outer probe wall upstream of the monolith. The boundary layer leads to a reduction of the velocity at the inlet

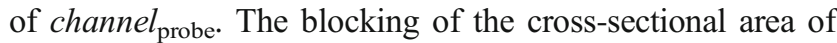
channel $_{\text {probe }}$ by the probe without boundary layer would lead to a smaller reduction of the volumetric flux by approx. $3.5 \%$ (ratio of the cross-sectional area of the probe and the channel). For a completely inserted probe, $V_{\text {channel,probe }}$ is reduced to approx. $55 \%$ of $V_{\text {channel,ref. }}$.

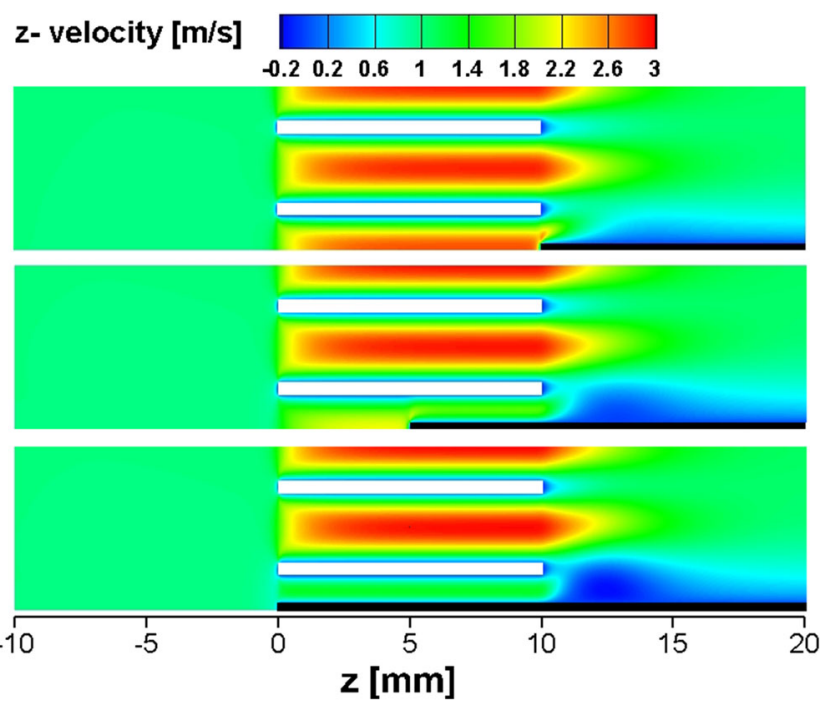

Fig. 8 Configuration center-downstream. Z-velocity in the $y z$-plane (view A-A in Fig. 3) for three positions $z_{\text {probe }}=10 / 5 / 0 \mathrm{~mm}$ of the probe tip (from top to bottom). The scale of the plots is compressed in the $z$ direction by a factor 3 


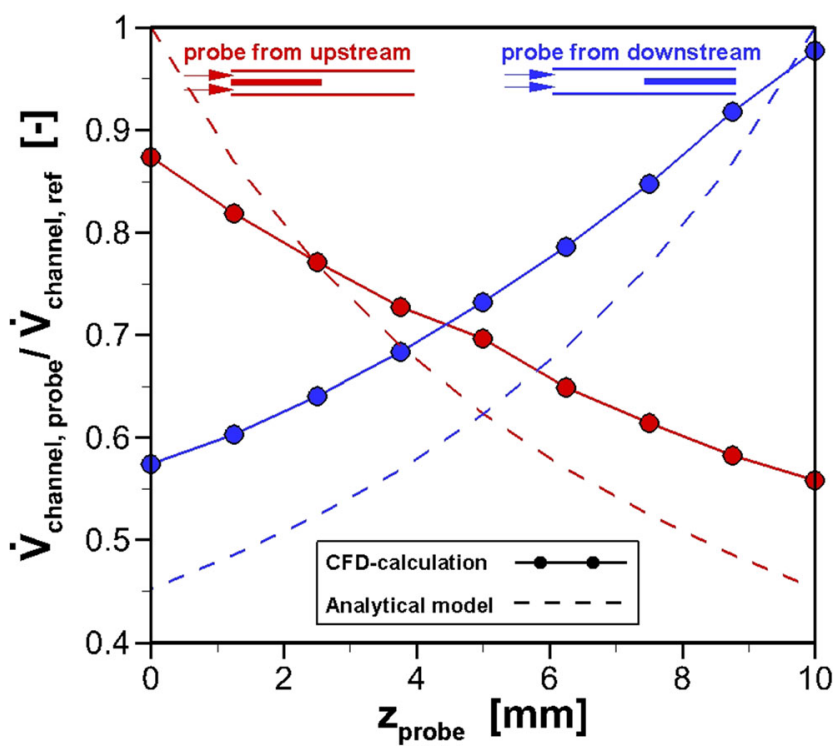

Fig. 9 Probe is in the center of the channel. Influence of axial probe position on the ratio of the volumetric fluxes of the channel with probe and a channel without probe $\dot{\boldsymbol{V}}_{\text {channel,probe }} / \dot{\boldsymbol{V}}_{\text {channel,ref. Comparison of }}$ CFD calculations and analytical model

For the configuration center-downstream, the dependencies are vice versa. At a position of the probe tip $z_{\text {probe }}=0$, the reduction of the volumetric flux is similar to center-upstream at $z_{\text {probe }}=10$. However, center-downstream at $z_{\text {probe }}=10 \mathrm{~mm}$ (probe is not inside the channel), the volumetric flux of channel $_{\text {probe }}$ is about $98 \%$ of the volumetric flux of channel $_{\text {ref. }}$ The reduction is smaller than in the configuration center-upstream at $z_{\text {probe }}=0 \mathrm{~mm}$. In configuration centerdownstream, the boundary layer at the surface of the probe is formed downstream of the monolith and shows a smaller influence on the volumetric flux in channel $_{\text {probe }}$ compared to configuration center-upstream. For center-upstream, the boundary layer at the probe tip is thicker at the inlet of channel $_{\text {probe }}$ than the boundary layer at the probe tip for center-downstream at the outlet of channel probe $_{\text {. }}$

In Fig. 9, the dashed lines show the results of the analytical model (see Sect. 4) representing the same qualitative behavior as the CFD calculations. The volumetric flux in channel $_{\text {probe }}$ is not reduced for the probe tip at the channel inlet (centerupstream at $z_{\text {probe }}=0 \mathrm{~mm}$ ) or at the channel outlet (centerdownstream at $z_{\text {probe }}=10 \mathrm{~mm}$ ) because the boundary layer at the probe wall outside of the channel is not taken into account in the model. The model overestimates the reduction of the volumetric flux in channel $l_{\text {probe }}$ for a completely inserted probe. This overestimation is a result of the wake region behind the outlet of channel $_{\text {probe, }}$ which influences the pressure field and the flow rate and which is not considered in the analytical model.

In the following, the flow field of the configuration cornerupstream will be discussed. Figure 10 shows isoplots of the velocity in the $z$-direction in a plane, which cuts the z-velocity [m/s]

$\begin{array}{lllllllll}-0.2 & 0.2 & 0.6 & 1 & 1.4 & 1.8 & 2.2 & 2.6 & 3\end{array}$
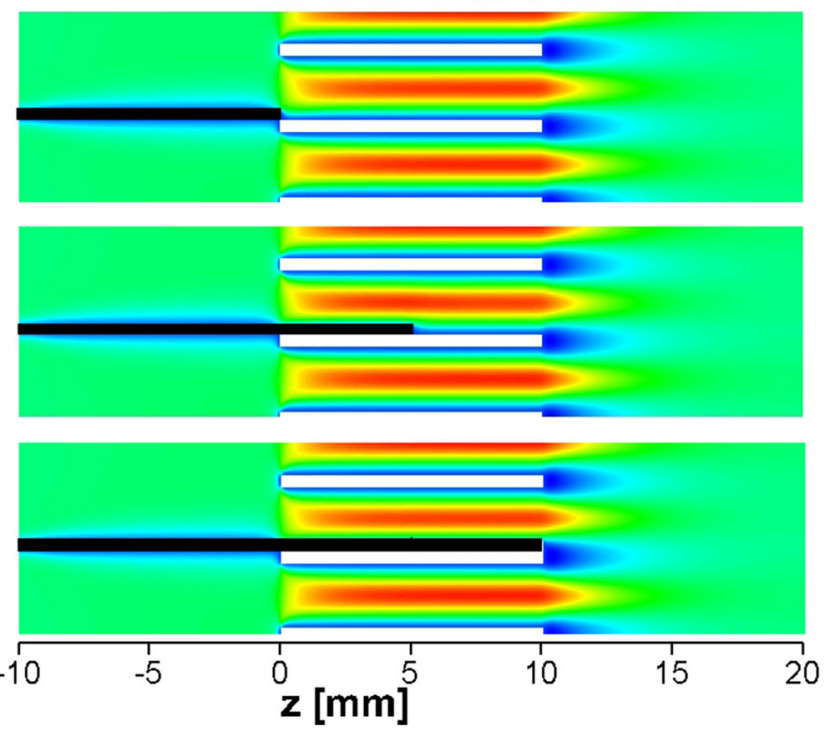

Fig. 10 Configuration corner-upstream. Z-velocity in the $y z$-plane (view A-A in Fig. 4) for three positions $z_{\text {probe }}=0 / 5 / 10 \mathrm{~mm}$ of the probe tip (from top to bottom). The scale of the plots is compressed in the $z$-direction by a factor 2

calculation domain diagonally, for three different positions of the probe tip (from top to bottom: $z_{\text {probe }}=0 / 5 / 10 \mathrm{~mm}$ ). Contrary to the configurations with the probe in the center of the channel, the whole channel $l_{\text {probe }}$ is now in the calculation domain (cross section A-A in Fig. 4). The influence of the probe on the velocity field is small. A similar behavior is observed for the configuration corner-downstream (not shown here).

In Fig. 11, the ratio of the volumetric fluxes $\dot{V}_{\text {channel,probe } / ~}$ $\dot{V}_{\text {channel,ref }}$ are plotted against the axial position for the configurations with the probe in the corner of the channel. For a completely inserted probe, $\dot{V}_{\text {channel,probe }} / \dot{V}_{\text {channel,ref }}$ is approx. $94 \%$. The direction of insertion does not influence this value. In the corner of the channel, the velocity decreases to zero towards the wall (see channel $_{\text {ref }}$ ). Therefore, the adhesion at the wall of the capillary shows only a small

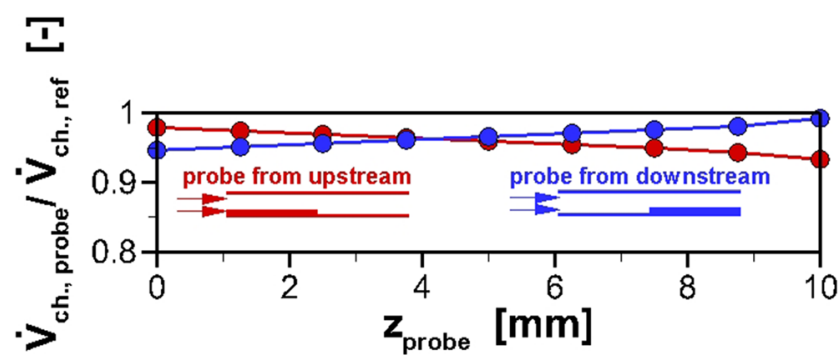

Fig. 11 Probe is in the corner of the channel. Influence of axial probe position on the ratio of the volumetric fluxes of the channel with probe and a channel without probe $\dot{V}_{\text {channel,probe }} / \dot{V}_{\text {channel,ref }}$ 


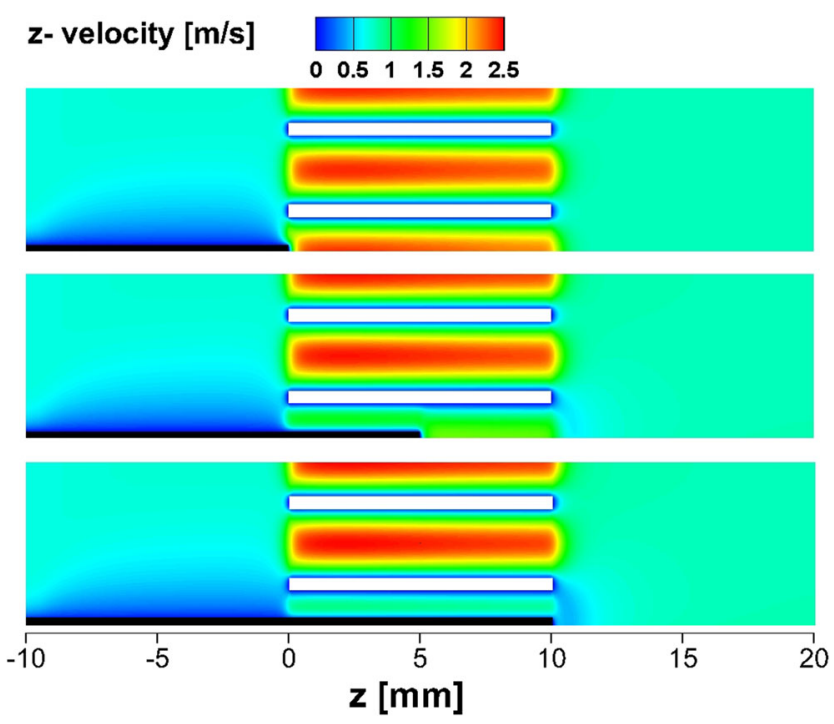

Fig. 12 Configuration center-upstream. Z-velocity in the $y z$-plane (view A-A in Fig. 3) for three positions $z_{\text {probe }}=0 / 5 / 10 \mathrm{~mm}$ of the probe tip (from top to bottom). The scale of the plots is compressed in the $z$-direction by a factor 3

effect on the overall velocity profile in channel $_{\text {probe }}$ as discussed previously [11].

On the surface of the probe, which is outside of the channel, a boundary layer forms similar to the one for the configurations with the probe in the center. However, the boundary layer in the corner configurations influences the flow field in the channel only slightly (flux reduction by approx. $2 \%$ ). In the configuration corner-upstream, the boundary layer is located partially upstream of the front wall of the solid region. In the configuration corner-downstream, it is partially located downstream of the rear wall of the solid region. Consequently, the ratio of volumetric fluxes in the cases where the probe is not in the channel (center-upstream at $z_{\text {probe }}=0 \mathrm{~mm}$ or center-downstream at $z$ probe $=10 \mathrm{~mm}$ ) is approx. $98 \%$. It is smaller than for the same axial positions in the center configurations.

\subsubsection{Calculation with Chemical Conversion}

For the results shown in this section, the boundary conditions of case b in Table 1 were used.

Firstly, the flow field for configuration center-upstream will be discussed. Figure 12 shows isoplots of the velocity in the $z$-direction in the $y z$-plane through the center of the channels (see cross section A-A in Fig. 3) for three different positions of the probe tip (from top to bottom: $z_{\text {probe }}=0 / 5 / 10 \mathrm{~mm}$ ). Again, the velocity in channel $l_{\text {probe }}$ is smaller the deeper the probe is inserted into the channel. The regions with reduced velocity downstream of the channel $_{\text {probe }}$ (wake regions), which can be identified in the flow without reaction, have nearly vanished and can be only identified for the case $z$ probe $=10 \mathrm{~mm}$. As the kinematic viscosity of the flow increases with temperature, the flow is dominated by viscous forces z- velocity $[\mathrm{m} / \mathrm{s}]$
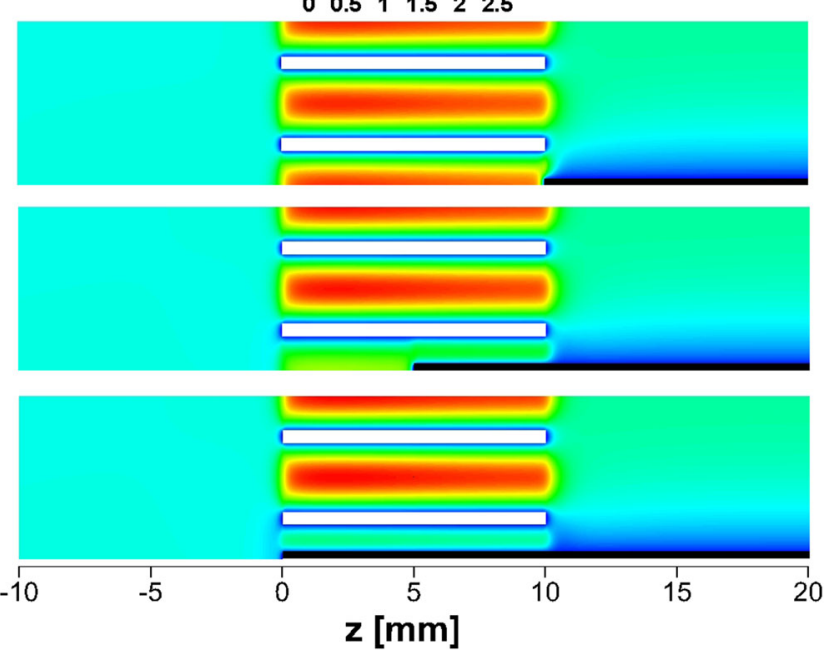

Fig. 13 Configuration center-downstream. Z-velocity in the $y z$-plane (view A-A in Fig. 3) for three positions $z_{\text {probe }}=10 / 5 / 0 \mathrm{~mm}$ of the probe tip (from top to bottom). The scale of the plots is compressed in the $z$-direction by a factor 3

compared to the case without reaction. The Reynolds number in the cases with reaction is $R e \approx 10$, whereas in the "cold" flow it is $R e \approx 80$.

In Fig. 13, the velocity into the $z$-direction is shown for the configuration center-downstream for three different positions of the probe tip (from top to bottom: $z_{\text {probe }}=10 / 5 / 0 \mathrm{~mm}$ ). As already shown for the case without reaction, the results are qualitatively the same as those for the configuration centerupstream at the same depth of immersion of the probe.

In the calculations with chemical conversion, the gas phase temperature is lower than the wall temperature at the channel inlet. The gas phase temperature reaches the constant wall temperature at approx. $z=1 \mathrm{~mm}$. In the following, the mass fluxes in the channels are analyzed, as they are independent of temperature in contrast to the previously analyzed volumetric fluxes in case of isothermal flow. The ratios of the mass fluxes in channelprobe and channel $l_{\text {ref }} \dot{M}_{\text {channel,probe }} / \dot{M}_{\text {channel,ref }}$ are the same for the same depth of immersion of the probe independent of direction of insertion (Fig. 14). For a probe, which is fully inserted into the channel, the volumetric flux is reduced to approx. $48 \%$ of the flux of an empty channel.

The results of the analytical model (dashed lines) show a better agreement with the results of the CFD calculation for the case with chemical conversion compared with the case without chemical conversion (see Figs. 9 and 14). The flow field is dominated by viscous forces for the case with chemical conversion due to the higher temperature (smaller Reynolds number). Therefore, the presumptions made in the analytical model (fully developed flow field, no influence of the flow field outside of the monolith) match more closely the conditions in the case with reaction than in the case without chemical conversion. 


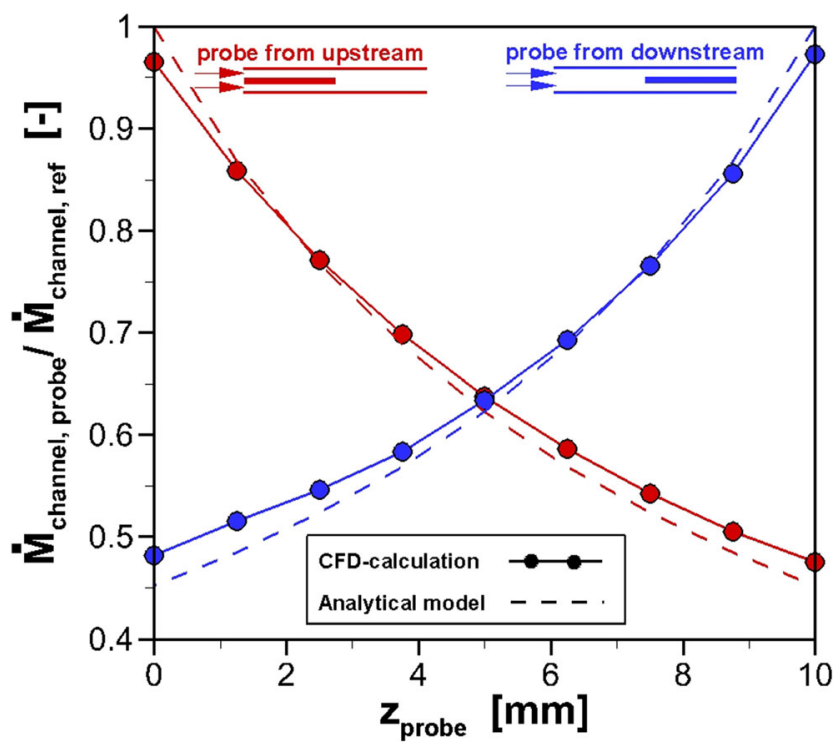

Fig. 14 Probe is in the center of the channel. Influence of axial probe position on the ratio of the mass fluxes $\dot{\boldsymbol{M}}_{\text {channel,probe }} / \dot{\boldsymbol{M}}_{\text {channel,ref }}$ of the channel with probe and a channel without probe. Comparison of CFD calculation and analytical model

In Fig. 15, the flow field of configuration corner-upstream is shown for three different positions of the probe tip. As already observed for the case without chemical conversion (Fig. 10), the velocity field is only slightly influenced by the probe. The results for configuration corner-downstream are not shown here, as the results are similar to the ones for corner-upstream and provide no new information.

\section{z-velocity $[\mathrm{m} / \mathrm{s}]$}
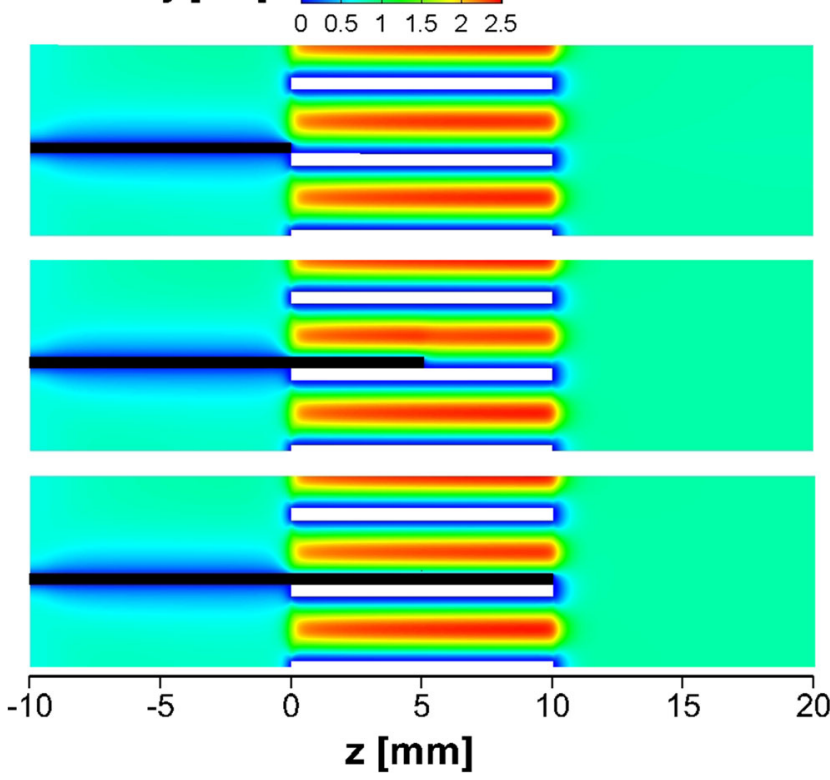

Fig. 15 Configuration corner-upstream. Z-velocity in the yz-plane (view A-A in Fig. 4) for three positions $z_{\text {probe }}=0 / 5 / 10 \mathrm{~mm}$ of the probe tip (from top to bottom). The scale of the plots is compressed in the $z$-direction by a factor 2

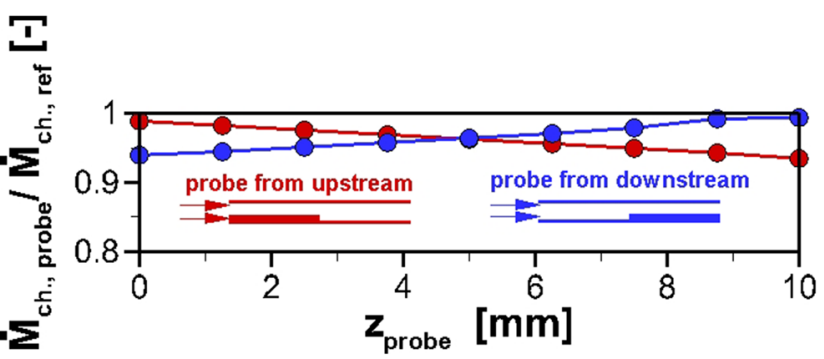

Fig. 16 Probe is in the corner of the channel. Influence of axial probe position on the ratio of the mass fluxes $\dot{\boldsymbol{M}}_{\text {channel,probe }} / \dot{\boldsymbol{M}}_{\text {channel,ref }}$ of the channel with probe and a channel without probe

The reduction of the mass fluxes $\dot{M}_{\text {channel,probe }}$ versus $\dot{M}_{\text {channel,ref }}$ (plotted in Fig. 16) differs only slightly from the results for the reduction of the volumetric fluxes for the case without reaction (see Fig. 11).

\subsection{Influence of Probe on Concentration Profiles}

\subsubsection{Impact of Suction}

All results shown in this section were obtained applying the boundary conditions of case $\mathrm{b}$ in Table 1 .

In Fig. 17, the values of the mole fractions of $\mathrm{CH}_{4}$ (educt), $\mathrm{H}_{2} \mathrm{O}$ (side product), and $\mathrm{H}_{2}$ (product) for configuration centerupstream with suction and without suction are shown. It is evident that the mole fractions are not affected by suction.

Figure 18 shows the same comparison for configuration corner-upstream. Again, the curves for the cases with and without suction agree. However, the distributions of the species at the probe tip are different compared to the center configuration (compare to Fig. 17). As will be discussed below, a radial gradient of the species mole fractions is observed in every channel. Thus, moving the probe in radial direction changes the gas composition at the probe tip.

The suction at the probe tip shows no influence on the species concentrations compared to the case without suction. Only a small portion of the volumetric flux of the entire channel is sucked into the capillary. Due to acceleration (area reduction and temperature increase), the mean velocity inside a channel without probe is approximately $u=1.3 \mathrm{~m} / \mathrm{s}$, which is equal to a volumetric flux of approx. $50 \mathrm{ml} / \mathrm{min}$. The volumetric flux sucked in at the probe tip was $2 \mathrm{ml} / \mathrm{min}$ in the calculations, similar to the value in the experiments.

\subsubsection{Comparison of Experiment and Calculation (CPOX)}

The results shown in this section were obtained applying the boundary conditions of case $\mathrm{c}$ in Table 1.

The distributions of the mole fractions of $\mathrm{CH}_{4}$ (top), $\mathrm{H}_{2} \mathrm{O}$ (middle), and $\mathrm{H}_{2}$ (bottom) in the $y z$-plane (view A-A in Fig. 3) 


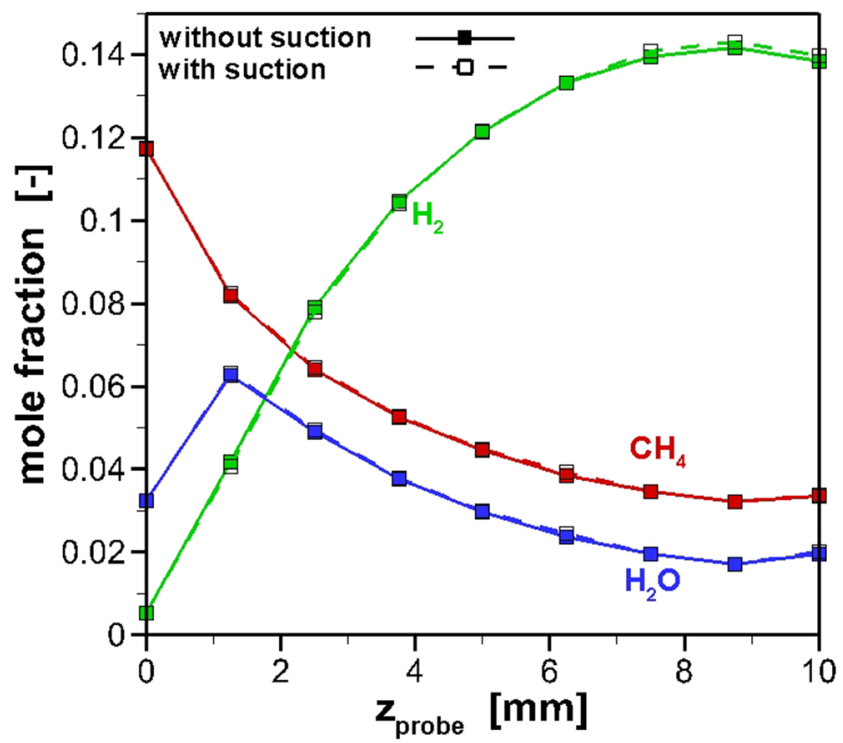

Fig. 17 Configuration center-upstream. Comparison of mole fractions of the species $\mathrm{CH}_{4}, \mathrm{H}_{2} \mathrm{O}$, and $\mathrm{H}_{2}$ for the cases without suction (full lines with filled symbols) and with suction (dashed lines with empty symbols)

for configuration center-upstream are plotted in Fig. 19. The probe tip is at $z_{\text {probe }}=10 \mathrm{~mm}$. We chose a small number of color levels for the depiction of the mole fractions to highlight the small differences within the distributions. In the short oxidation zone $(z=0-1 \mathrm{~mm})$, methane is consumed forming water and hydrogen. Farther downstream, the water is converted by steam reforming. The comparison of the mole fractions in the different channels shows that the reaction process is enhanced (in terms of the z-position) in the channel with probe than in a channel without probe because the residence time increases. Except for the first millimeter, only small radial

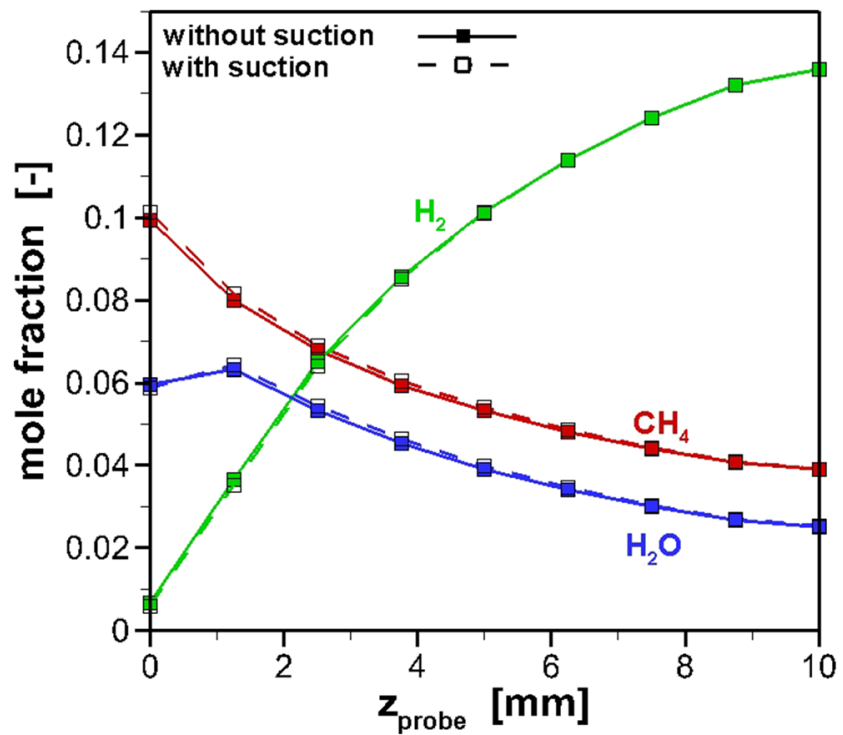

Fig. 18 Configuration corner-upstream. Comparison of mole fractions of the species $\mathrm{CH}_{4}, \mathrm{H}_{2} \mathrm{O}$, and $\mathrm{H}_{2}$ as a function of axial coordinate for the cases without suction (full lines with filled symbols) and with suction (dashed lines with empty symbols)

\section{$\mathrm{CH}_{4}$ mole fraction [-]}

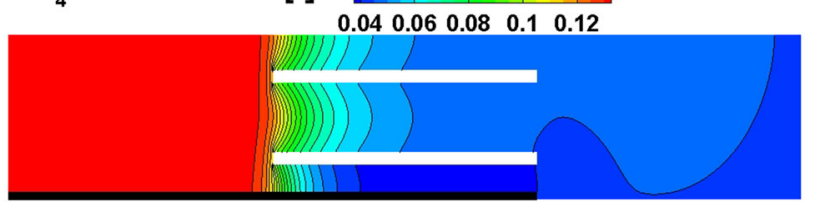

\section{$\mathrm{H}_{2} \mathrm{O}$ mole fraction [-]}

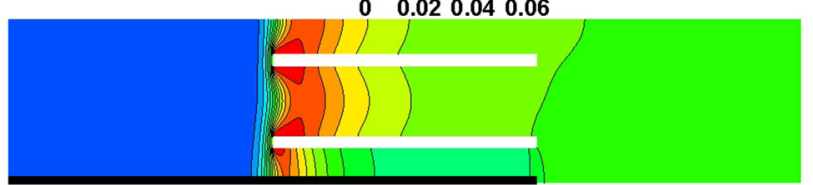

$\mathrm{H}_{2}$ mole fraction [-]

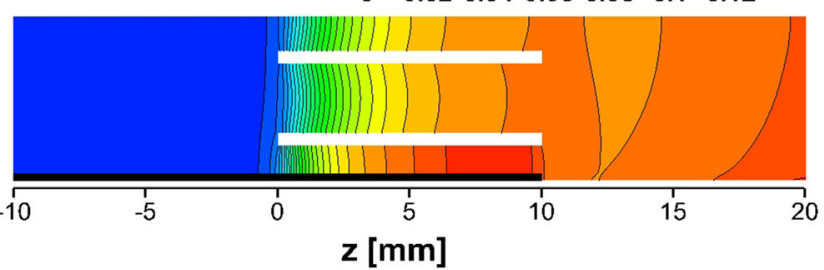

Fig. 19 Configuration center-upstream (probe tip at $z_{\text {probe }}=10 \mathrm{~mm}$ ). Flow direction is from left to right. Mole fractions of $\mathrm{CH}_{4}$ (top), $\mathrm{H}_{2} \mathrm{O}$ (middle), and $\mathrm{H}_{2}$ (bottom) in the yz-plane (view A-A in Fig. 3). The scale of the plots is compressed in the $z$-direction by a factor 3

gradients are observed in each channel. External mass transfer limitation of the reaction rate is restricted to the entrance zone.

In Fig. 20, the measured data for $\mathrm{CPOX}$ of $\mathrm{CH}_{4}$ at $\mathrm{C} / \mathrm{O}=1.0$ (square symbols) is plotted together with the calculated mole fraction profiles starting in front of the catalyst at $z=-2.5 \mathrm{~mm}$

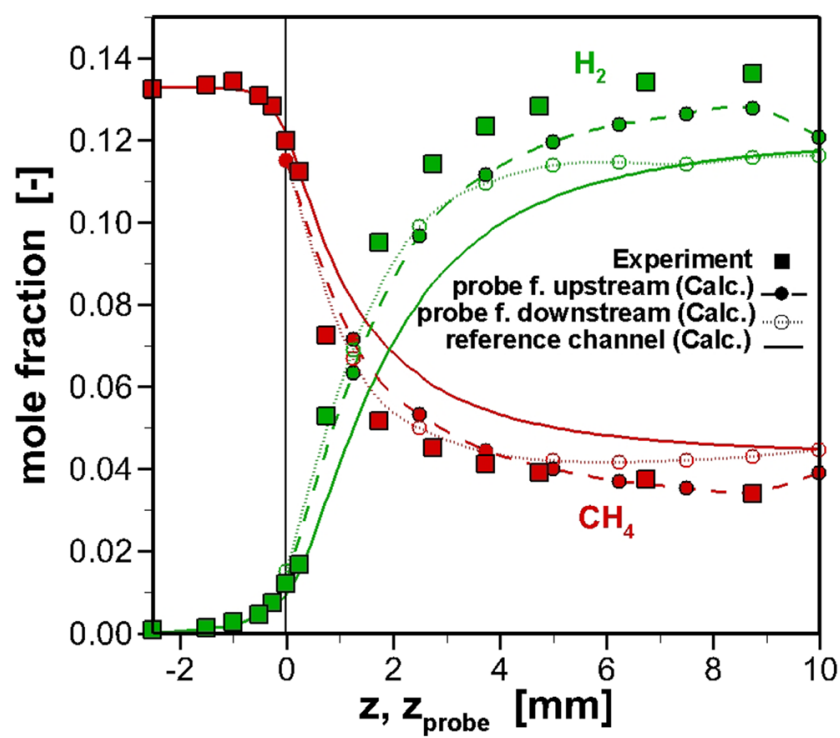

Fig. 20 Probe is in the center of the channel. Boundary case "c": temperature profile at the wall. Comparison of calculated and measured mole fractions of the species $\mathrm{CH}_{4}$ and $\mathrm{H}_{2}$ as a function of axial coordinate for CPOX. Square symbols: measurement, full lines: values in channel $_{\text {ref }}$ (without probe), dashed lines with filled circles: values in channel $_{\text {probe }}$ (probe enters from upstream), dotted lines with empty circles: values in channel $_{\text {probe }}$ (probe enters from downstream) 
up to the end at $z=10 \mathrm{~mm}$. The full lines depict the values along the channel axis for channel $l_{\text {ref. }}$ The dashed lines show the values for different positions of the probe tip in channel $_{\text {probe }}$ for configuration center-upstream. The dotted lines show the respective values for configuration center-downstream. For the clarity of the picture, the values for the mole fraction of water are omitted. The experimental results on the partial oxidation of methane over Rh have been discussed in our previous papers $[11,28]$. For this reason, no stand-alone discussion for the experimental results is given herein.

The mole fractions of methane and hydrogen start to change approx. $2 \mathrm{~mm}$ upstream of the catalyst. As stated above, gas phase reactions can be neglected. Thus, molecular diffusion is the only source for the decrease of reactants and the increase of products in front of the catalyst, both in the experiment and in the simulation (see also [11]). The operating conditions in the region between $-1<z<1 \mathrm{~mm}$, e.g., high temperature $(T=873 \mathrm{~K})$ and large concentration gradients, favor diffusion effects. In this zone, the diffusive flux of species is in the same range of magnitude as the convective flux.

Inside the monolith $(z=0-10 \mathrm{~mm})$, the mole fraction of $\mathrm{H}_{2}$ at an axial z-position in channel $_{\text {probe }}$ is larger compared to the value in channel $_{\text {ref }}$ for the identical axial position. According to the reaction stoichiometry, the mole fractions of $\mathrm{CH}_{4}$ are smaller. As discussed before, the influence of the probe leads to a reduction of the volumetric flux, an increase of residence time of the mixture, and an increase in reaction progress in the channel with probe versus an empty channel.

In the first section of the catalyst $(z=0-4 \mathrm{~mm})$, the influence of the capillary on the profiles is smaller for configuration center-upstream (dashed lines) compared to configuration center-downstream (dotted lines). The insertion length of the probe for a distinct position $z_{\text {probe }}$ is larger for the case center-downstream compared to the case center-upstream. The larger the insertion length, the smaller is the volumetric flux. In the second section of the catalyst $(z=4-10 \mathrm{~mm})$, influence of the capillary on the profiles is vice versa, because the insertion length of the probe inside the channel is larger for case center-upstream compared to case center-downstream.

For the probe tip at the outlet of the channel at $z$ probe $=10 \mathrm{~mm}$, the mole fractions in the channels with and without probe approach each other. The molecular diffusion perpendicular to and along the flow direction in the outlet region leads to a mixture of the species. This characteristic can also be identified in Fig. 19. The effect was not present in the calculations shown in [11], as the calculation domain ended at $z=10 \mathrm{~mm}$. In the measurements, this behavior is not distinct. The diffusion between neighboring channels at the outlet of the monolith is limited due to the presence of the back heat shield, which touches the catalyst in the experiments [9].

Generally, the calculated mole fractions of the species in channel $_{\text {probe }}$ agree better with the measured data than the values in an empty reference channel. However, the position of the probe in the $x y$-plane within the individual channel is not clearly defined in the experiments. Overall, the results for the calculations with configuration center-upstream agree better with the measured data than the results for the configuration center-downstream. In our experiments, the probe enters the catalyst from upstream.

For the configuration center-downstream, the results in the second half of channel $_{\text {probe }}$ agree better with the values inside channel $_{\text {ref }}$ Considering this region, it could be beneficial to conduct experiments with the probe entering the catalyst from downstream. However, the difficulty concerning the avoidance of gas phase reactions inside the capillary would still apply.

In Fig. 21, the measured data for CPOX (square symbols) is plotted together with the calculated mole fraction profiles starting in front of the catalyst at $z=-2.5 \mathrm{~mm}$ up to the end at $z=10 \mathrm{~mm}$. The dashed lines show the values for configuration corner-upstream, the dotted lines the values for configuration corner-downstream. For the discussion of the results, it is important to mention that the values in the empty channel channel $_{\text {ref }}$ (full lines) are extracted at the same distance to the channel corner as in the channel $_{\text {probe }}$. For all positions of the probe tip $z_{\text {probe }}>1.25 \mathrm{~mm}$, the mole fractions in the channels with probe agree very well with the values in the reference channel. The impact of the probe on the volumetric flux is weak for a corner configuration (see Fig. 11). A larger deviation can be found for the position $z_{\text {probe }}=0 \mathrm{~mm}$. For corner-downstream, the mole fraction of methane remains at the entrance level as no surface reaction can take place at the wall, which is in contact with the probe tip. In contrast, the mole fraction at the same position in channel $_{\text {ref }}$ decreases due to reaction. For corner-downstream, the mole fraction of methane is below the value of the reference channel. The velocity directly upstream of the probe tip is very small, leading to a larger residence time of the mixture and to a locally higher conversion of methane. Differences between the mole fractions of hydrogen directly at the entrance cannot be identified. The larger diffusion coefficient of this species leads to a homogenization of the profile in radial and axial direction.

For the corner configuration, all results of the calculations differ from the measured data. The position of the probe in the $x y$-plane is not clearly defined in our experiments. We believe the position of the capillary is in between the center and the wall of the channel and bent by gravity.

\subsubsection{Analytical Model for Correction of Concentration Values}

The results shown in Fig. 22 were obtained applying the boundary conditions of case $\mathrm{b}$ in Table 1 . The temperature of the wall is constant and forces the gas phase temperature to the same temperature within a short distance. 


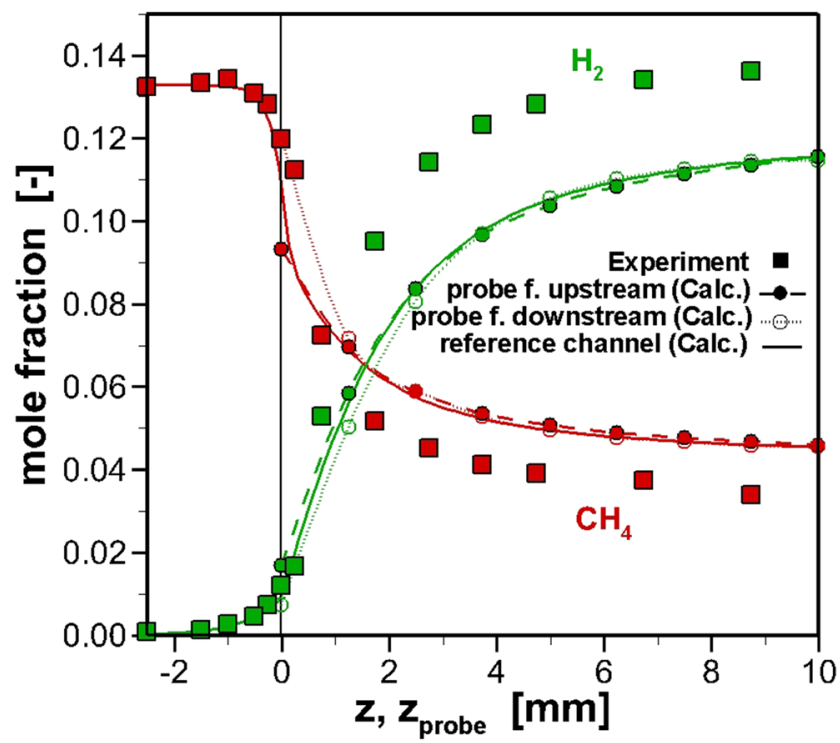

Fig. 21 Probe is in the corner of the channel. Boundary case "c": temperature profile at the wall. Comparison of calculated and measured mole fractions of the species $\mathrm{CH}_{4}$ and $\mathrm{H}_{2}$ as a function of axial coordinate for CPOX. Square symbols: measurement, full lines: values in channel $_{\text {ref }}$ (without probe), dashed lines with filled circles: values in channel $_{\text {probe }}$ (probe enters from upstream), dotted lines with empty circles: values in channel $_{\text {probe }}$ (probe enters from downstream)

Therefore, the volumetric flux in each channel is nearly constant along the $z$-coordinate.

Figure 22 shows the mole fractions of two species sucked from channel $l_{\text {probe }}$ at different axial positions of the probe tip $z_{\text {probe }}$ (dashed lines with empty symbols) and the concentrations at equivalent positions $z$ at the axis of a channel without probe channel $_{\text {ref }}$ (full lines). For each axial position $z_{\text {probe }}$, the mole fraction of $\mathrm{H}_{2}$ is larger and the mole fraction of $\mathrm{CH}_{4}$ is smaller than at $z_{\text {ref }}$. Hence, in an equivalent experiment, the data collected on the basis of suction in channel probe $_{\text {en }}$ would be defective. The farther downstream the location of the tip is, the more overrated the progress of conversion at each tip position would be.

Applying Eq. (14) for each position $z_{\text {probe }}$ in channel $_{\text {probe }}$, a position $z$ in channel $l_{\text {ref }}$ can be found for which the residence times are the same (see Sect. 4). The filled symbols in Fig. 22 show the values of the concentration calculated with Eq. (14). The corrected values agree very well with the values in channel $l_{\text {ref. }}$ In principal, this approach offers the possibility to correct the bias of experimental data introduced by a suction probe with the configuration center-upstream. However, a constant wall temperature is a requirement.

As shown in Fig. 23, the correction works not as well for case $\mathrm{c}$ in Table 1, in which a temperature profile along the wall was applied. The analytical model is based on the assumption that the volumetric flux inside a single channel is constant. This assumption becomes invalid for a temperature change of the mixture. Nevertheless, the application of the correction function leads to a smaller difference between

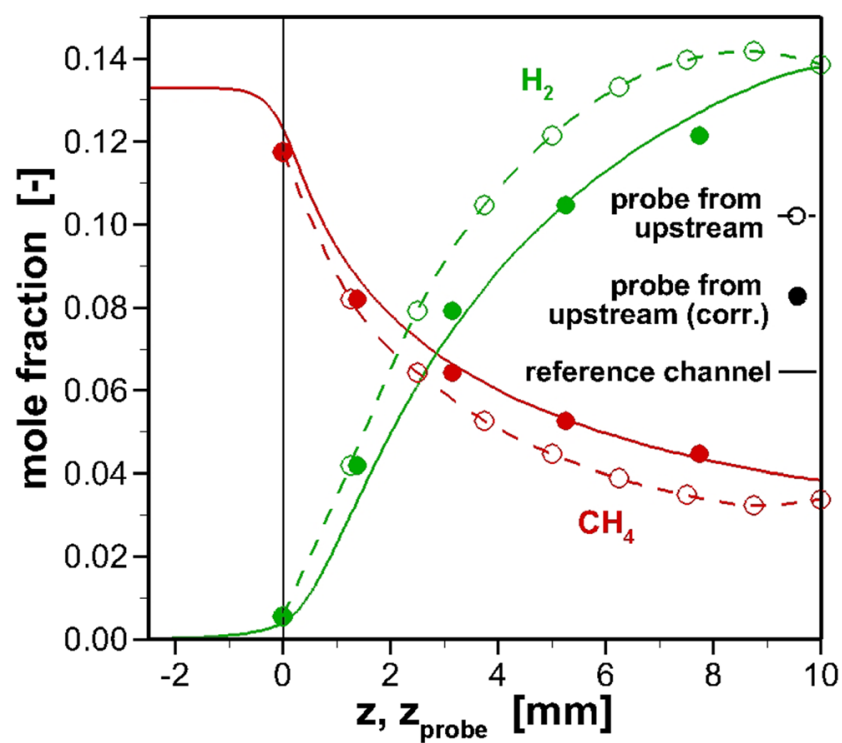

Fig. 22 Configuration corner-upstream. Boundary case "b": temperature of wall is constant. Comparison of mole fractions of the species $\mathrm{CH}_{4}$ and $\mathrm{H}_{2}$ as a function of axial coordinate. Values of channel $_{\text {ref: full lines, values }}$

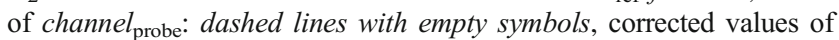
channel $_{\text {probe: filled symbols }}$

the values in channel $l_{\text {probe }}$ and in channel $_{\text {ref }}$ than without correction.

\subsubsection{Comparison of Experiment and Calculation (CO-OX)}

All simulation results shown in this section were obtained applying the boundary conditions of case $\mathrm{d}$ in Table 1 .

The measured data for CO-OX (square symbols) is plotted in Fig. 24 together with the calculated mole fraction profiles. The region from $z=-2.5 \mathrm{~mm}$ (upstream of catalyst) to $z=10 \mathrm{~mm}$ (catalyst outlet) is shown. Again, the full lines depict the values along the channel axis for channel $_{\text {ref. }}$ The dashed lines show the values for configuration centerupstream. The dotted lines show the values for configuration center-downstream.

As the experimental results for the oxidation of carbon monoxide on palladium are presented herein for the first time, a short discussion may be given even though the experiments serve mainly as an exemplary case for the simulations. For these experimental results, it has to be taken into account that the $\mathrm{CO}$ and $\mathrm{CO}_{2}$ concentrations are $\leq 1087 \mathrm{ppm}$. The procedure during the detection of the concentration profiles is not designed for these small concentrations. The sample $(2 \mathrm{ml} /$ min), which is sucked from the channel, is diluted with $1000 \mathrm{ml} / \mathrm{min}_{2}$ to reach the necessary flow for the analytics. The small concentrations of $\leq 1087 \mathrm{ppm}$ in the sample are thus further decreased and are close to the detection limit of the used analytics.

Low concentration of $\mathrm{CO}$ was chosen based on exhaust gas after treatment. Additionally, we aimed at a constant temperature over the length of the catalyst to eliminate this 


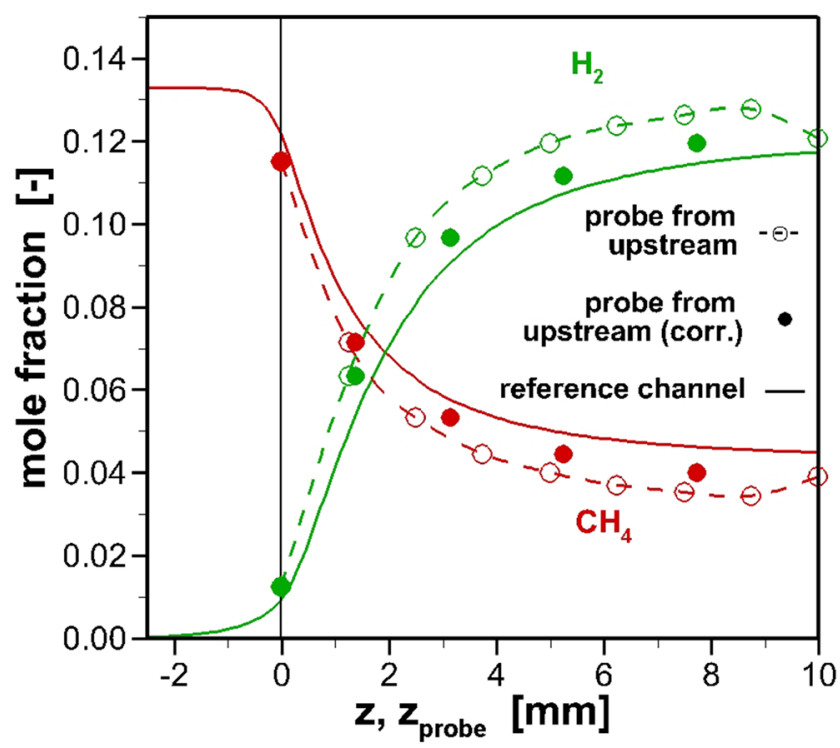

Fig. 23 Configuration corner-upstream. Boundary case "c": temperature profile at the wall. Comparison of mole fractions of the species $\mathrm{CH}_{4}$ and $\mathrm{H}_{2}$ as a function of axial coordinate. Values of channel $_{\text {ref: full lines, values }}$ of channel $l_{\text {probe: }}$ dashed lines with empty symbols, corrected values of channel $_{\text {probe: }}$ filled symbols

parameter for the CFD simulations. This goal was reached, as only an increase of temperature by $10 \mathrm{~K}$ from catalyst inlet $(z=0 \mathrm{~mm})$ to catalyst outlet $(z=10 \mathrm{~mm})$ was detected (not shown). In the measurements, complete conversion of $\mathrm{CO}$ is observed. However, due to the small concentrations of $\mathrm{CO}$ for $z \geq 3 \mathrm{~mm}$, the exact point at which complete conversion is reached cannot be distinguished.

The simulations reveal that the process takes place earlier (in terms of the $z$-position) in the channel with probe than in a channel without probe. The conversion in the calculations is complete at approx. $z=4 \mathrm{~mm}$ for the channel with probe. As a result, the calculated mole fractions in the different channels are the same for $z>6 \mathrm{~mm}$. The direction of insertion of the probe (insertion from upstream or downstream) has a negligible influence on the results. The comparison to the measurements shows that the reaction progress is too fast on the first $2 \mathrm{~mm}$ in the calculations for channel $_{\text {ref }}$ as well as channel $l_{\text {probe }}$. This deviation might be caused by the reaction mechanism applied.

The findings for CO-OX reveal the same trend for the comparison for the channel with probe and a channel without probe as observed for CPOX (compare Fig. 20). However, the influence of the direction of insertion, which is visible for CPOX in the second half of the catalyst $(z>5 \mathrm{~mm})$, cannot be observed due to complete conversion for CO-OX.

\section{Summary}

The application of suction probe techniques to collect spatially resolved gas phase concentration profiles in catalytic monoliths is widely used today. In the paper, the impact of the

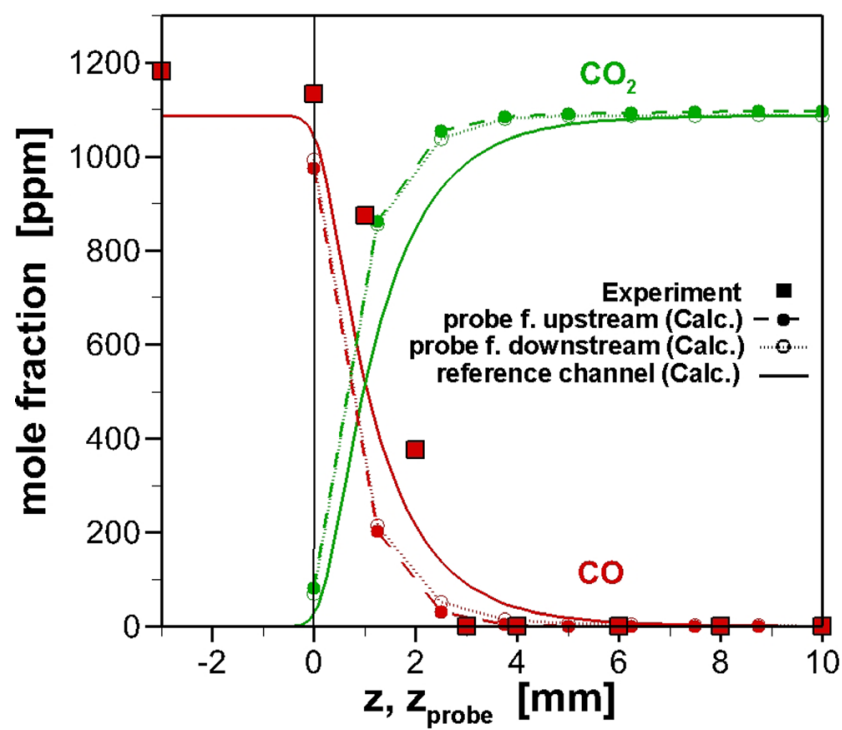

Fig. 24 Probe is in the center of the channel. Comparison of calculated and measured mole fractions of the species $\mathrm{CO}$ and $\mathrm{CO}_{2}$ as a function of axial coordinate for CO-OX. Square symbols: measurement, full lines: values in channel $_{\text {ref }}$ (without probe), dashed lines with filled circles: values in channel probe (probe enters from upstream), dotted lines with empty circles: values in channel $_{\text {probe }}$ (probe enters from downstream)

capillary on the measured data is investigated by means of CFD simulations.

The results document that the use of a suction probe for the measuring inside monolithic honeycomb structures can have a significant impact on the profiles. In general, the volumetric flux in a channel with capillary is reduced. Compared to the equivalent axial position of an empty channel, the residence time at the probe tip is larger. The increased residence time leads to faster reaction progress and an axial shift of the concentration profiles upstream compared to an empty channel. A simple analytical model can predict the reduction of the mass flux due to the insertion of the probe for the flow with reaction. A capillary, which is completely inserted into the channel and positioned in the center of the channel, can cause a reduction of volumetric flux of over $50 \%$, although the probe blocks only $3.5 \%$ of the channel. This result is independent of the direction of insertion.

For the CPOX of methane, the influence on the concentration profiles is slightly smaller in the first half of the channel for a configuration with the probe entering from upstream of the catalyst than for one with the probe entering from downstream. In contrast, the bias of the data is clearly smaller in the second half of the channel, for the probe entering from downstream. Therefore, in this region, a smaller influence on measured data is to be expected compared to the configuration in which the probe enters from upstream. However, gas phase reactions of the sampled gas flow in the capillary might occur for large temperatures of the system (e.g., for $\mathrm{CPOX}$ ). For a configuration with the capillary 
positioned in a corner of a channel, the corruption of data is negligible. The only region for which a difference is observed is the inlet of the channel. In this region, mass transfer limitation of the reaction rate and radial gradients has to be taken into account. The suction at the probe tip showed no influence on the mixture field in all investigated cases.

For the oxidation of carbon monoxide, the probe also leads to an axial shift of the concentration profiles compared to a channel without probe. Compared to the measured data, the complete conversion of $\mathrm{CO}$ is reached farther upstream (smaller $z$-position) for the calculations.

The analysis of the flow fields reveal that the characteristics are qualitatively the same for a distinct position of the probe tip, independent from the direction of insertion of the capillary. Especially for the calculations at low temperature (without reaction and for $\mathrm{CO}-\mathrm{OX}$ ), a wake region can be identified downstream of the channel with probe. The extent of this wake region decreases with decreasing Reynolds number. The molecular diffusion leads to an axial smearing of the profiles near the inlet of the monolith and to a mixing of the fluids of the channel with probe with neighboring channels near the outlet.

The influence of the probe on the measured concentration profiles has to be considered for interpretation of measured data. CFD simulations as well as analytical deductions support this conclusion.

Acknowledgments The authors would like to thank Delphi Corp. and Umicore AG \& Co. KG for providing the model catalysts for the presented experimental research. We would like to acknowledge financial support by the Helmholtz Association.

Compliance with ethical standards The authors declare that they have no competing interests.

\section{References}

1. Zellner, A., Suntz, R., Deutschmann, O.: Two-dimensional spatial resolution of concentration profiles in catalytic reactors by planar laser-induced fluorescence: no reduction over diesel oxidation catalysts. Angew. Chem. Int. Ed. 54, 2653 (2015)

2. Schneider, A., Mantzaras, J., Bombach, R., Schenker, S., Tylli, N., Jansohn, P.: Laser induced fluorescence of formaldehyde and Raman measurements of major species during partial catalytic oxidation of methane with large $\mathrm{H} 2 \mathrm{O}$ and $\mathrm{CO} 2$ dilution at pressures up to 10 bar. Proc. Combust. Inst. 31, 1973 (2007)

3. Partridge, W.P., Storey, J.M., Lewis,S., Smithwick, R., DeVault, G.L., Cunningham, M.J., Currier, N., Yonushonis, T.M.: Time-resolved measurements of emission transients by mass spectrometry. SAE Technical paper, 2000-01-2952 (2000)

4. Sa, J., Fernandes, D.L.A., Aiouache, F., Goguet, A., Hardacre, C., Lundie, D., Naeem, W., Partridge, W.P., Stere, C.: SpaciMS: spatial and temporal operando resolution of reactions within catalytic monoliths. Analyst 135, 2260 (2010)
5. Chan, D., Tischer, S., Heck, J., Diehm, C., Deutschmann, O.: Correlation between catalytic activity and catalytic surface area of a Pt/A12O3 DOC: an experimental and microkinetic modeling study. Appl. Catal. B Environ. 156-157, 153 (2014)

6. Bugosh, G.S., Easterling, V.G., Rusakova, I.A., Harold, M.P.: Anomalous steady-state and spatio-temporal features of methane oxidation on $\mathrm{Pt} / \mathrm{Pd} / \mathrm{A} 12 \mathrm{O} 3$ monolith spanning lean and rich conditions. Appl. Catal. B Environ. 165, 68 (2015)

7. Horn, R., Williams, K.A., Degenstein, N.J., Bitsch-Larsen, A., Nogare, D.D., Tupy, S.A., Schmidt, L.D.: Methane catalytic partial oxidation on autothermal $\mathrm{Rh}$ and Pt foam catalysts: oxidation and reforming zones, transport effects, and approach to thermodynamic equilibrium. J. Catal. 249, 380 (2007)

8. Donazzi, A., Livio, D., Maestri, M., Beretta, A., Groppi, G., Tronconi, E., Forzatti, P.: Synergy of homogeneous and heterogeneous chemistry probed by in situ spatially resolved measurements of temperature and composition. Angew. Chem. Int. Ed. 50, 3943 (2011)

9. Livio, D., Diehm, C., Donazzi, A., Beretta, A., Deutschmann, O.: Catalytic partial oxidation of ethanol over Rh/A12O3: spatially resolved temperature and concentration profiles. Appl. Catal. A 467, 530 (2013)

10. Choi, J.-S., Partridge, W.P., Daw, C.S.: Spatially resolved in situ measurements of transient species breakthrough during cyclic, lowtemperature regeneration of a monolithic $\mathrm{Pt} / \mathrm{K} / \mathrm{Al} 2 \mathrm{O} 3 \mathrm{NOx}$ storagereduction catalyst. Appl. Catal. A Gen. 293, 24 (2005)

11. Hettel, M., Diehm, C., Torkashvand, B., Deutschmann, O.: Critical evaluation of in situ probe techniques for catalytic honeycomb monoliths. Catal. Today 216, 2 (2013)

12. Diehm, C., Deutschmann, O.: Hydrogen production by catalytic partial oxidation of methane over staged $\mathrm{Pd} / \mathrm{Rh}$ coated monoliths: spatially resolved concentration and temperature profiles. Int. J. Hydrog. Energy 39, 17998 (2014)

13. Song, X., Parker, G.G., Johnson, J.H., Naber, J.D., Pihl, J.A.: A SRC model calibration approach with spatially resolved measurements and NH3 storage distributions. Emiss. Control Sci. Technol. 1, 98 (2015)

14. ANSYS $®$ Academic Research, Release 13.0, ANSYS, Inc

15. Kee, R.J., Coltrin, M.E., Glarborg, P.: Chemically reacting flow: theory and practice. Wiley, Hoboken (2003)

16. Deutschmann, O.: Modeling and simulation of heterogeneous catalytic reactions: from the molecular process to the technical system. Wiley, VCH Weinheim (2011)

17. Bissett, E.J.: An asymptotic solution for wachcoat pore diffusion in catalytic monoliths. Emiss. Control Sci. Technol. 1, 3 (2015)

18. Deutschmann, O., Schwiedernoch, R., Maier, L.I., Chatterjee, D.: In: Iglesia, J.J.S. E., Fleisch, T.H. (eds.) Studies in Surface Science and Catalysis, Vol. 136, Elsevier, p. 251. 2 (2001)

19. Beretta, A., Donazzi, A., Livio, D., Maestri, M., Groppi, G., Tronconi, E., Forzatti, P.: Optimal design of a CH4 CPO-reformer with honeycomb catalyst: combined effect of catalyst load and channel size on the surface temperature profile. Catal. Today 171, 79 (2011)

20. Bitsch-Larsen, A., Horn, R., Schmidt, L.D.: Catalytic partial oxidation of methane on rhodium and platinum: spatial profiles at elevated pressure. Appl. Catal. A Gen. 348, 165 (2008)

21. Veser, G., Frauhammer, J.: Modelling steady state and ignition during catalytic methane oxidation in a monolith reactor. Chem. Eng. Sci. 55, 2271 (2000)

22. Deutschmann, O., Schmidt, L.D.: Modeling the partial oxidation of methane in a shortcontact-time reactor. AICHE J. 44, 2465 (1998)

23. Hauck, C., Tischer, S., Maier, L., Deutschmann, O.: Estimation and modeling of local aging effects of three-way-catalysts by analysis of their spatial temperature and CO conversion profiles. Can. J. Chem. Eng. 92, 1587 (2014) 
24. Hettel, M., Diehm, C., Deutschmann, O.: Answer to the comment from Goguet et al. to the paper "the critical evaluation of in situ probe techniques for catalytic honeycomb monoliths" by Hettel et al. [1]. Catal. Today 236, 209 (2014)

25. Incropera, F., DeWitt, D.: Fundamentals of Heat and Mass Transfer. Wiley, Hoboken (2006)

26. Siegloch, H.: Technische Fluidmechanik. VDI-Verlag, Germany (1996)
27. Gossler, H., Kee, B.L., Zhu, H., Hettel, M., Deutschmann, O., Kee, R.J.: Flow and pressure characteristics in rectangular channels with internal cylindrical bodies. Chem. Eng. Sci. 149, 296 (2016)

28. Hettel, M., Diehm, C., Bonart, H., Deutschmann, O.: Numerical simulation of a structured catalytic methane reformer by DUO: the new computational interface for OpenFOAM ${ }^{\circledR}$ and DETCHEM $^{\mathrm{TM}}$. Catal. Today 258, 230 (2015) 